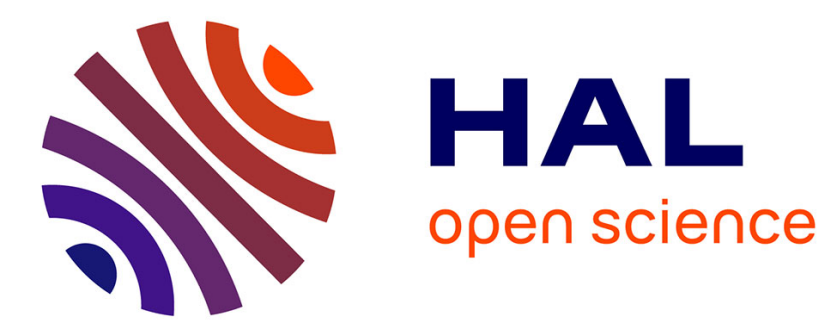

\title{
Towards More Precise Rewriting Approximations
} Yohan Boichut, Jacques Chabin, Pierre Réty

\section{To cite this version:}

Yohan Boichut, Jacques Chabin, Pierre Réty. Towards More Precise Rewriting Approximations. Proceedings of Language and Automata Theory and Applications (LATA), Mar 2015, Nice, France. hal-01135918

\section{HAL Id: hal-01135918 https://hal.science/hal-01135918}

Submitted on 25 Oct 2021

HAL is a multi-disciplinary open access archive for the deposit and dissemination of scientific research documents, whether they are published or not. The documents may come from teaching and research institutions in France or abroad, or from public or private research centers.
L'archive ouverte pluridisciplinaire HAL, est destinée au dépôt et à la diffusion de documents scientifiques de niveau recherche, publiés ou non, émanant des établissements d'enseignement et de recherche français ou étrangers, des laboratoires publics ou privés.

\section{다(1) $\$$}

Distributed under a Creative Commons Attribution - NonCommercial| 4.0 International 
Version of Record: https://www.sciencedirect.com/science/article/pii/S0022000017300065 Manuscript_9943b6fea105bb6bb15d89ffba1a6cde

\title{
Towards more Precise Rewriting Approximations ${ }^{\dagger}$
}

\author{
Yohan Boichut $^{\mathrm{a}, *}$, Jacques Chabin $^{\mathrm{a}}$, Pierre Réty $^{\mathrm{a}}$ \\ ${ }^{a}$ LIFO - Université d'Orléans, B.P. 6759, 45067 Orléans cedex 2, France
}

\begin{abstract}
To check a system, some verification techniques consider a set of terms $I$ that represents the initial configurations of the system, and a rewrite system $R$ that represents the system behavior. To check that no undesirable configuration is reached, they compute an over-approximation of the set of descendants (successors) issued from $I$ by $R$, expressed by a tree language. Their success highly depends on the quality of the approximation. Some techniques have been presented using regular tree languages, and more recently using non-regular languages to get better approximations: using context-free tree languages [1] on the one hand, using synchronized tree languages [2] on the other hand. In this paper, we merge these two approaches to get even better approximations: we compute an over-approximation of the descendants, using synchronized-contextfree tree languages expressed by logic programs. We give several examples for which our procedure computes the descendants in an exact way, whereas the former techniques compute a strict over-approximation.

Keywords: term rewriting, tree languages, logic programming, reachability, rewriting approximations.

\footnotetext{
${ }^{*}$ Principal corresponding author.

Email addresses: yohan.boichut@univ-orleans.fr (Yohan Boichut),

$\dagger$ A preliminary version of this paper appeared in the Proceedings of the 9th International
}

jacques.chabin@univ-orleans.fr (Jacques Chabin), pierre.rety@univ-orleans.fr (Pierre Réty) Conference on Language and Automata Theory and Applications (LATA), LNCS 8977, 2015.
\end{abstract}




\section{Introduction}

To check systems like cryptographic protocols or Java programs, some verification techniques consider a set of terms $I$ that represents the initial configurations of the system, and a rewrite system $R$ that represents the system behavior $[3,4,5]$. To check that no undesirable configuration is reached, they compute an over-approximation of the set of descendants ${ }^{2}$ (successors) issued from $I$ by $R$, expressed by a tree language. Let $R^{*}(I)$ denote the set of descendants of $I$, and consider a set Bad of undesirable terms. Thus, if a term of $B a d$ is reached from $I$, i.e. $R^{*}(I) \cap B a d \neq \emptyset$, it means that the protocol or the program is flawed. In general, it is not possible to compute $R^{*}(I)$ exactly. Instead, one computes an over-approximation $A p p$ of $R^{*}(I)$ (i.e. $A p p \supseteq R^{*}(I)$ ), and checks that $A p p \cap B a d=\emptyset$, which ensures that the protocol or the program is correct.

However, I, Bad and App have often been considered as regular tree languages, recognized by finite tree automata. In the general case, $R^{*}(I)$ is not regular, even if $I$ is. Moreover, the expressiveness of regular languages is poor. Then the over-approximation App may not be precise enough, and we may have $A p p \cap B a d \neq \emptyset$ whereas $R^{*}(I) \cap B a d=\emptyset$. In other words, the protocol is correct, but we cannot prove it. Some work has proposed CEGAR-techniques (CounterExample Guided Approximation Refinement) to conclude as often as possible $[3,6,7]$. However, in some cases, no regular over-approximation works [8].

To overcome this theoretical limit, the idea is to use more expressive languages to express the over-approximation, i.e. non-regular ones. However, to be able to check that $A p p \cap B a d=\emptyset$, we need a class of languages closed under intersection and whose emptiness is decidable. Actually, if we assume that Bad is regular, closure under intersection with a regular language is enough. The class of context-free tree languages has these properties, and an approximation technique using context-free tree languages has been proposed in [1]. On the

\footnotetext{
${ }^{2}$ I.e. terms obtained by applying arbitrarily many rewrite steps on the terms of $I$.
} 
other hand, the class of synchronized tree languages [9] also has these properties, and an approximation technique using synchronized tree languages has been proposed in [2]. Both classes include regular languages, but they are incomparable. Context-free tree languages cannot express dependencies between different branches, except in some cases, whereas synchronized tree languages cannot express vertical dependencies.

We want to use a more powerful class of languages that can express the two kinds of dependencies together: the class of synchronized-context-free tree(tuple) languages $[10,11]$, which has the same properties as context-free languages and as synchronized languages, i.e. closure under union, closure under intersection with a regular language, decidability of membership and emptiness.

In this paper, we propose a procedure that always terminates and that computes an over-approximation of the descendants obtained by a linear rewrite system, using synchronized-context-free tree-(tuple) languages expressed by logic programs. Compared to our previous work [2], we introduce "input arguments" in predicate symbols, which is a major technical change that highly improves the quality of the approximation, and that requires new results and new proofs. This work is a first step towards a verification technique offering more than regular approximations. Some on-going work is discussed in Section 6 in order to make this technique be an acceptable verification technique.

The paper is organized as follows. Term rewriting and synchronized-contextfree tree languages are introduced in Section 2. Then technical results needed in the sequel are established in Section 3. Our main contribution, i.e. computing approximations, is presented in Section 4. Finally, in Section 5 our technique is applied to examples, in particular when $R^{*}(I)$ can be expressed in an exact way neither by a context-free language, nor by a synchronized language.

Comparison with $[\mathbf{1}, \mathbf{2}, \mathbf{3}, \mathbf{4}, \mathbf{5}]$ : If no input arguments are used, this paper is equivalent to [2], which actually also needed the rewrite system to be leftand-right linear (the erratum of [2] is given in [12]), due to the semantics of logic programs. An extension of [2] to deal with non right-linear rewrite system 
is proposed in [13].

If no input arguments are used, predicate symbols have only one argument, and assuming some additional restrictions, the logic program can be viewed as a finite tree automaton (predicate symbols are considered as states), which then generates a regular language. In this particular case, the procedure presented in this paper works as the tree automaton completion of $[3,4,5]$. However, $[3,4,5]$ only need left-linearity, and [3] uses a set of equation $E$ as an heuristics for guiding the approximation, whereas our procedure does not because equations do not make sense when working with predicate symbols with several arguments, i.e. with tree-tuples. On the other hand, our procedure always terminate, but $[3,4,5]$ could also always terminate if a bound for the number of states was fixed.

The completion procedure of [1] computes an over-approximation of the descendants using a context-free tree language defined by an indexed linear tree grammar (ILTG). Actually, ILTGs look like and are equi-expressive with (top-down) pushdown tree automata. The rewrite system is assumed to be leftlinear, and the procedure always terminate. Roughly speaking, when rewriting a term $t$ generated by the current grammar (to get the descendants of $t$ ), the substitution (which is a match) is stored into the stack. However, to make the stack alphabet finite, substitutions are pruned, which amounts to merge various substitutions. Compared to our procedure, [1] is more automated, because it does not need additional heuristics to guide the approximation. However, it is limited by the use of context-free tree languages. Unfortunately, it is difficult to compare our procedure with that of [1], because they are quite different and use different formalisms.

Other Related Work: The class of tree-tuples whose overlapping coding is recognized by a tree automaton on the product alphabet [14] (called "regular tree relations" by some authors), is strictly included in the class of rational tree 
relations [15]. The latter is equivalent to the class of non-copying ${ }^{3}$ synchronized languages [16], which is strictly included in the class of synchronized languages.

Context-free tree languages (i.e. without assuming a particular strategy for grammar derivations) [17] are equivalent to OI (outside-in strategy) context-free tree languages, but are incomparable with IO (inside-out strategy) context-free tree languages $[18,19]$. The IO class (and not the OI one) is strictly included in the class of synchronized-context-free tree languages. The latter is equivalent to the "term languages of hyperedge replacement grammars", which are equivalent to the tree languages definable by attribute grammars [20, 21]. However, we prefer to use the synchronized-context-free tree languages, which use the well known formalism of pure logic programming, for its implementation ease.

Much other work computes the descendants in an exact way using regular tree languages (in particular the recent paper [22]). In general the set of descendants is not regular even if the initial set is. Consequently strong restrictions over the rewrite system are needed to get regular descendants, which are not suitable in the framework of protocol or program verification.

\section{Preliminaries}

Consider a finite ranked alphabet $\Sigma=\{a, b, f, g, h, \ldots\}$ and a set of variables $\operatorname{Var}=\{x, y, z, \ldots\}$. Each symbol $f \in \Sigma$ has a unique arity, denoted by $\operatorname{ar}(f)$. The notions of first-order term, position and substitution are defined as usual. Given $\sigma$ and $\sigma^{\prime}$ two substitutions, $\sigma \circ \sigma^{\prime}$ denotes the substitution such that for any variable $x, \sigma \circ \sigma^{\prime}(x)=\sigma\left(\sigma^{\prime}(x)\right) . T_{\Sigma}$ denotes the set of ground terms (without variables) over $\Sigma$. For a unary functional symbol $f, \overbrace{f(f(\ldots f(t))}^{n \text { times }})$ is denoted by $f^{n}(t)$. For a term $t, \operatorname{Var}(t)$ is the set of variables of $t, \operatorname{Pos}(t)$ is the set of positions of $t$. For $p \in \operatorname{Pos}(t), t(p)$ is the symbol of $\Sigma \cup \operatorname{Var}$ occurring at position $p$ in $t$, and $\left.t\right|_{p}$ is the subterm of $t$ at position $p$. The term $t$ is linear if each variable of $t$ occurs only once in $t$. The term $t\left[t^{\prime}\right]_{p}$ is obtained from $t$ by replacing

\footnotetext{
${ }^{3}$ Clause heads are assumed to be linear.
} 
the subterm at position $p$ by $t^{\prime} . \operatorname{Pos} \operatorname{Var}(t)=\{p \in \operatorname{Pos}(t) \mid t(p) \in \operatorname{Var}\}$,

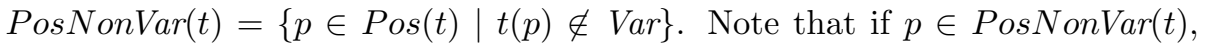
$\left.t\right|_{p}=f\left(t_{1}, \ldots, t_{n}\right)$, and $i \in\{1, \ldots, n\}$, then $p . i$ is the position of $t_{i}$ in $t$. For $p, p^{\prime} \in \operatorname{Pos}(t), p<p^{\prime}$ means that $p$ occurs in $t$ strictly above $p^{\prime}$. Let $t, t^{\prime}$ be terms, $t$ is more general than $t^{\prime}$ (denoted $t \leq t^{\prime}$ ) if there exists a substitution $\rho$ s.t. $\rho(t)=t^{\prime}$. Let $\sigma, \sigma^{\prime}$ be substitutions, $\sigma$ is more general than $\sigma^{\prime}$ (denoted $\left.\sigma \leq \sigma^{\prime}\right)$ if there exists a substitution $\rho$ s.t. $\rho \circ \sigma=\sigma^{\prime}$.

A rewrite rule is an oriented pair of terms, written $l \rightarrow r$. We always assume that $l$ is not a variable, and $\operatorname{Var}(r) \subseteq \operatorname{Var}(l)$. A rewrite system $R$ is a finite set of rewrite rules. lhs stands for left-hand-side, rhs for right-hand-side. The rewrite relation $\rightarrow_{R}$ is defined as follows: $t \rightarrow_{R} t^{\prime}$ if there exist a position

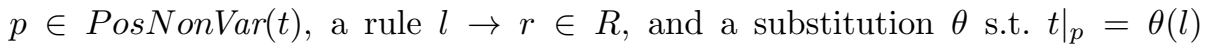
and $t^{\prime}=t[\theta(r)]_{p} . \quad \rightarrow_{R}^{*}$ denotes the reflexive-transitive closure of $\rightarrow_{R} . \quad t^{\prime}$ is a descendant of $t$ if $t \rightarrow_{R}^{*} t^{\prime}$. If $E$ is a set of ground terms, $R^{*}(E)$ denotes the set of descendants of elements of $E$. The rewrite rule $l \rightarrow r$ is left (resp. right) linear if $l$ (resp. $r$ ) is linear. $R$ is left (resp. right) linear if all its rewrite rules are left (resp. right) linear. $R$ is linear if $R$ is both left and right linear.

In the following, we consider the framework of pure logic programming, and the class of synchronized-context-free tree-tuple ${ }^{4}$ languages $[10,11]$, which is presented as an extension of the class of synchronized tree-tuple languages defined by CS-clauses [9, 23]. Given a set Pred of predicate symbols; atoms, goals, bodies and Horn-clauses are defined as usual. Note that both goals and bodies are sequences of atoms. We will use letters $G$ or $B$ for sequences of atoms, and $A$ for atoms. Given a goal $G=A_{1}, \ldots, A_{k}$ and positive integers $i, j$, we define $\left.G\right|_{i}=A_{i}$ and $\left.G\right|_{i . j}=\left.\left(A_{i}\right)\right|_{j}=t_{j}$ where $A_{i}=P\left(t_{1}, \ldots, t_{n}\right)$. Let $G$ be a sequence of atoms, $A$ an atom occuring in $G$ and $B$ a new atom. We denote by $G[A \leftarrow B]$ the replacement of the atom $A$ by $B$ in $G$.

Definition 1. The tuple of terms $\left(t_{1}, \ldots, t_{n}\right)$ is flat if $t_{1}, \ldots, t_{n}$ are variables.

\footnotetext{
${ }^{4}$ For simplicity, "tree-tuple" is sometimes omitted.
} 
The sequence of atoms $B$ is flat if for each atom $P\left(t_{1}, \ldots, t_{n}\right)$ of $B,\left(t_{1}, \ldots, t_{n}\right)$ is flat. $B$ is linear if each variable occurring in $B$ (possibly at subterm position) occurs only once in B. Note that the empty sequence of atoms (denoted by $\emptyset$ ) is flat and linear.

$A$ Horn clause $P\left(t_{1}, \ldots, t_{n}\right) \leftarrow B$ is:

- empty if $P\left(t_{1}, \ldots, t_{n}\right)$ is flat, i.e. $\forall i \in\{1, \ldots, n\}, t_{i}$ is a variable.

- normalized if $\forall i \in\{1, \ldots, n\}, t_{i}$ is a variable or contains only one occurrence of function-symbol. A program is normalized if all its clauses are normalized.

Example 1. Let $x, y, z$ be variables. The sequence of atoms $P_{1}(x, y), P_{2}(z)$ is flat, whereas $P_{1}(x, f(y)), P_{2}(z)$ is not flat. The clause $P(x, y) \leftarrow Q(x, y)$ is empty and normalized. The clause $P(f(x), y) \leftarrow Q(x, y)$ is normalized whereas $P(f(f(x)), y) \leftarrow Q(x, y)$ is not.

Definition 2. A logic program with modes is a logic program such that a modetuple $\vec{m} \in\{I, O\}^{n}$ is associated to each predicate symbol $P$ ( $n$ is the arity of $P$ ). In other words, each predicate argument has mode $I$ (Input) or $O$ (Output). To distinguish them, output arguments will be covered by a hat.

Notation: Let $P$ be a predicate symbol. $\operatorname{Ar} \operatorname{In}(P)$ is the number of input $\operatorname{arguments}$ of $P$, and $\operatorname{ArOut}(P)$ is the number of output arguments. Let $B$ be a sequence of atoms (possibly containing only one atom). $\operatorname{In}(B)$ is the input part of $B$, i.e. the tuple composed of the input arguments of $B . \operatorname{Ar} \operatorname{In}(B)$ is the arity of $\operatorname{In}(B) . \operatorname{Var}^{i n}(B)$ is the set of variables that appear in $\operatorname{In}(B)$. $\operatorname{Out}(B), \operatorname{ArOut}(B)$, and $\operatorname{Var}^{\text {out }}(B)$ are defined in a similar way. We also define $\operatorname{Var}(B)=\operatorname{Var}^{\text {in }}(B) \cup \operatorname{Var}^{\text {out }}(B)$.

Example 2. Let $B=P\left(\widehat{t_{1}}, \widehat{t_{2}}, t_{3}\right), Q\left(\widehat{t_{4}}, t_{5}, t_{6}\right)$. Then, $\operatorname{Out}(B)=\left(t_{1}, t_{2}, t_{4}\right)$ and $\operatorname{In}(B)=\left(t_{3}, t_{5}, t_{6}\right)$.

Definition 3. Let $B=A_{1}, \ldots, A_{n}$ be a sequence of atoms. We say that $A_{j} \succ A_{k}$ (possibly $j=k$ ) if $\exists y \in \operatorname{Var}^{i n}\left(A_{j}\right) \cap \operatorname{Var}^{\text {out }}\left(A_{k}\right)$. In other words an 
input of $A_{j}$ depends on an output of $A_{k}$. We say that $B$ has a loop if $A_{j} \succ^{+} A_{j}$ for some $A_{j}\left(\succ^{+}\right.$is the transitive closure of $\left.\succ\right)$.

Example 3. $Q(\widehat{x}, s(y)), R(\widehat{y}, s(x))$ (where $x, y$ are variables) has a loop because $Q(\widehat{x}, s(y)) \succ R(\widehat{y}, s(x)) \succ Q(\widehat{x}, s(y))$.

Definition 4. A Synchronized-Context-Free (S-CF) program Prog is a logic program with modes, whose clauses $H \leftarrow B$ satisfy:

- In $(H)$. Out $(B)$ (. is the tuple concatenation) is a linear tuple of variables, i.e. each tuple-component is a variable, and each variable occurs only once, - and $B$ does not have a loop.

A clause of an S-CF program is called $S$-CF clause.

Example 4. $\operatorname{Prog}=\{P(\widehat{x}, y) \leftarrow P(\widehat{s(x)}, y)\}$ is not an S-CF program because $\operatorname{In}(H) \cdot \operatorname{Out}(B)=(y, s(x))$ is not a tuple of variables. $\operatorname{Prog}^{\prime}=\left\{P^{\prime}(\widehat{s(x)}, y) \leftarrow\right.$ $\left.P^{\prime}(\widehat{x}, s(y))\right\}$ is an S-CF program because $\operatorname{In}(H) \cdot \operatorname{Out}(B)=(y, x)$ is a linear tuple of variables, and there is no loop in the clause body.

Definition 5. Let Prog be an S-CF program. Given a predicate symbol $P$ without input arguments, the tree-(tuple) language generated by $P$ is $L_{P r o g}(P)=$ $\left\{\vec{t} \in\left(T_{\Sigma}\right)^{\operatorname{ArOut}(P)} \mid P(\vec{t}) \in \operatorname{Mod}(\operatorname{Prog})\right\}$, where $T_{\Sigma}$ is the set of ground terms over the signature $\Sigma$ and $\operatorname{Mod}(\operatorname{Prog})$ is the least Herbrand model of Prog. $L_{\text {Prog }}(P)$ is called Synchronized-Context-Free language ( $S$-CF language).

Example 5. Let us consider the S-CF program without input arguments Prog $=$ $\left.\left\{P_{1}(\widehat{g(x, y)}) \leftarrow P_{2}(\widehat{x}, \widehat{y}) . P_{2}\left(\widehat{c(x, y)}, c \widehat{c x^{\prime}, y^{\prime}}\right)\right) \leftarrow P_{2}\left(\widehat{x}, \widehat{y^{\prime}}\right), P_{2}\left(\widehat{y}, \widehat{x^{\prime}}\right) . P_{2}(\widehat{a}, \widehat{a}) \leftarrow.\right\}$. The language generated by $P_{1}$ is $L_{\text {Prog }}\left(P_{1}\right)=\left\{g\left(t, t_{\text {sym }}\right) \mid t \in T_{\left\{c{ }^{2}, a \backslash 0\right\}}\right\}$, where $t_{\text {sym }}$ is the symmetric tree of $t$ (for instance $c(c(a, a), a)$ is the symmetric of $c(a, c(a, a)))$. This language is synchronized, but it is not context-free.

Example 6. $\operatorname{Prog}=\left\{S(\widehat{c(x, y)}) \leftarrow P(\widehat{x}, \widehat{y}, a, b) . P\left(\widehat{f(x)}, \widehat{g(y)}, x^{\prime}, y^{\prime}\right) \leftarrow P(\widehat{x}, \widehat{y}\right.$, $\left.\left.h\left(x^{\prime}\right), i\left(y^{\prime}\right)\right) . \quad P(\widehat{x}, \widehat{y}, x, y) \leftarrow\right\}$ is an S-CF program. The language generated by $S$ is $L_{\text {Prog }}(S)=\left\{c\left(f^{n}\left(h^{n}(a)\right), g^{n}\left(i^{n}(b)\right)\right) \mid n \in \mathbb{N}\right\}$, which is not synchronized (there are vertical dependencies) nor context-free. 
S-CF languages are closed under union, intersection, and emptiness is decidable [11]. Emptiness is linear in the number of clauses of the S-CF program. On the other hand, consider a S-CF program Prog" that computes the intersection of a S-CF program Prog with a regular program Prog'. Let Pred, Pred', Pred" be the set of predicate symbols of Prog, Prog $^{\prime}$, Prog ${ }^{\prime \prime}$ respectively. A bound for the size of Pred" is $\mid$ Pred $^{\prime \prime}|\leq|$ Pred $\mid .2^{\text {ArMax(Pred).|Pred }}{ }^{\prime}$, where ArMax(Pred) is the biggest arity of the elements of Pred.

Definition 6. The clause $H \leftarrow B$ is non-copying if the tuple $\operatorname{Out}(H) \cdot \operatorname{In}(B)$ is linear. An S-CF program is non-copying if all its clauses are non-copying.

Example 7. The clause $P(\widehat{d(x, x)}, y) \leftarrow Q(\widehat{x}, p(y))$ is copying. $P(\widehat{c(x)}, y) \leftarrow Q(\widehat{x}, p(y))$ is non-copying.

Remark: An S-CF program without input arguments is actually a CS-program (composed of CS-clauses) [9], which generates a synchronized language ${ }^{5}$. A noncopying normalized CS-program such that every predicate symbol has only one argument is called regular program. It is equivalent to a finite tree automaton. Indeed, clauses are of the form $P_{0}\left(f\left(\widehat{x_{1}, \ldots,} x_{n}\right)\right) \leftarrow P_{1}\left(\widehat{x_{1}}\right), \ldots, P_{n}\left(\widehat{x_{n}}\right)$, which is equivalent to the transition $f\left(P_{1}, \ldots, P_{n}\right) \rightarrow P_{0}$ where $P_{0}, P_{1}, \ldots, P_{n}$ are considered as states. Consequently it generates a regular tree language. Conversely, every regular tree language can be generated by a regular program.

Given an S-CF program, we focus on two kinds of derivations.

Definition 7. Given an $S$-CF program Prog and a sequence of atoms $G$,

- $G$ derives into $G^{\prime}$ by a resolution step if there exists a clause ${ }^{6} H \leftarrow B$ in Prog and an atom $A \in G$ such that $A$ and $H$ are unifiable by the most general unifier $\sigma$ (then $\sigma(A)=\sigma(H))$ and $G^{\prime}=\sigma(G)[\sigma(A) \leftarrow \sigma(B)]$. It is written $G \sim_{\sigma} G^{\prime}$.

\footnotetext{
${ }^{5}$ Initially, synchronized languages were presented using constraint systems (sorts of grammars) [24], and later using logic programs. CS stands for "Constraint System".

${ }^{6}$ We assume that the clause and $G$ have distinct variables.
} 
We consider the transitive closure $\sim^{+}$and the reflexive-transitive closure $\sim^{*}$ of $\leadsto$. If $G_{1} \sim_{\sigma_{1}} G_{2}$ and $G_{2} \sim_{\sigma_{2}} G_{3}$, we write $G_{1} \sim_{\sigma_{2} \circ \sigma_{1}}^{*} G_{3}$.

- $G$ rewrites into $G^{\prime}$ (possibly in several steps) if $G \sim_{\sigma}^{*} G^{\prime}$ s.t. $\sigma$ does not instantiate the variables of $G$. It is written $G \rightarrow_{\sigma}^{*} G^{\prime}$.

Example 8. Prog $=\left\{P\left(\widehat{x_{1}}, \widehat{g\left(x_{2}\right)}\right) \leftarrow P^{\prime}\left(\widehat{x_{1}}, \widehat{x_{2}}\right) . P\left(\widehat{f\left(x_{1}\right)}, \widehat{x_{2}}\right) \leftarrow P^{\prime \prime}\left(\widehat{x_{1}}, \widehat{x_{2}}\right) \cdot\right\}$, and consider $G=P(f(x), y)$. We have $P(f(x), y) \sim_{\sigma_{1}} P^{\prime}\left(f(x), x_{2}\right)$ with $\sigma_{1}=$ $\left[x_{1} / f(x), y / g\left(x_{2}\right)\right]$ and $P(f(x), y) \rightarrow_{\sigma_{2}} P^{\prime \prime}(x, y)$ with $\sigma_{2}=\left[x_{1} / x, x_{2} / y\right]$.

In the remainder of the paper, given an S-CF program Prog and two sequences of atoms $G_{1}$ and $G_{2}, G_{1} \sim_{\text {Prog }}^{*} G_{2}$ (resp. $G_{1} \rightarrow_{\text {Prog }}^{*} G_{2}$ ) also denotes that $G_{2}$ can be derived (resp. rewritten) from $G_{1}$ using clauses of Prog. Note that for any atom $A$, if $A \rightarrow B$ then $A \leadsto B$. On the other hand, $A \sim_{\sigma} B$ implies $\sigma(A) \rightarrow B$. Consequently, if $A$ is ground, $A \leadsto B$ implies $A \rightarrow B$.

It is well known that resolution is complete.

Theorem 1. Let $A$ be a ground atom. $A \in \operatorname{Mod}(\operatorname{Prog})$ iff $A \sim_{\text {Prog }}^{*} \emptyset$.

\section{Technical Lemmas}

Before describing in Section 4 our technique for computing non-regular approximations, we need some technical lemmas for proving our results.

Lemma 1. Let $t$ and $t^{\prime}$ be two terms such that $\operatorname{Var}(t) \cap \operatorname{Var}\left(t^{\prime}\right)=\emptyset$. Suppose that $t^{\prime}$ is linear. Assuming that $t$ and $t^{\prime}$ are unifiable, let $\sigma$ be the most general unifier of $t$ and $t^{\prime}$. Then, one has: $\forall x, y:(x, y \in \operatorname{Var}(t) \wedge x \neq y) \Rightarrow \operatorname{Var}(\sigma(x)) \cap$ $\operatorname{Var}(\sigma(y))=\emptyset$ and $\forall x: x \in \operatorname{Var}(t) \Rightarrow \sigma(x)$ is linear.

For the next lemmas, we introduce two notions allowing the extraction of variables occurring once in a sequence of atoms.

Definition 8. Let $G$ be a sequence of atoms. $\operatorname{Var}_{\text {Lin }}^{i n}(G)$ is a tuple of variables occurring in $\operatorname{In}(G)$ and not in $\operatorname{Out}(G)$, and $\operatorname{Var}_{\text {Lin }}^{\text {out }}(G)$ is a tuple of variable occurring in $\operatorname{Out}(G)$ and not in $\operatorname{In}(G)$. In both cases, tuples of variables are 
built in such a way that the number of occurences of variables is preserved for the concerned variables i.e. if a variable occurs $n$ times in $\operatorname{Var}_{\text {Lin }}^{i n}(G)$ (resp. $\left.\operatorname{Var}_{\text {Lin }}^{\text {out }}(G)\right)$ then the same variable occurs $n$ times in $\operatorname{In}(G)(\operatorname{resp}$. Out $(G))$.

Example 9. Let $\left.G=P\left(g\left(\widehat{f\left(x^{\prime}, z^{\prime}\right.}\right)\right), y^{\prime}\right), Q\left(\widehat{v^{\prime}}, g\left(z^{\prime}\right)\right)$. Then $\operatorname{Var}_{\text {Lin }}^{i n}(G)=\left(y^{\prime}\right)$ and $\operatorname{Var}_{\text {Lin }}^{\text {out }}(G)=\left(x^{\prime}, v^{\prime}\right)$.

Note that for a matter of simplicity, we denote by $x \in \operatorname{Var}_{\text {Lin }}^{i n}(G)$ (resp. $\left.x \in \operatorname{Var}_{\text {Lin }}^{\text {out }}(G)\right)$ that $x$ occurs in the tuple $\operatorname{Var}_{\text {Lin }}^{\text {in }}(G)\left(\operatorname{resp} . \operatorname{Var}_{\text {Lin }}^{\text {out }}(G)\right)$. The following lemma focuses on a property of a sequence of atoms obtained after a resolution step.

Lemma 2. Let Prog be a non-copying $S$-CF program, and $G$ be a sequence of atoms such that $\operatorname{Out}(G)$ is linear, $\operatorname{In}(G)$ is linear and $G$ does not contain loops. We assume $e^{7}$ that variables occurring in Prog are different from those occurring in $G$. If $G \leadsto_{\sigma} G^{\prime}$, then $G^{\prime}$ is loop free, $\sigma\left(\operatorname{Var}_{\text {Lin }}^{\text {in }}(G)\right)$.Out $\left(G^{\prime}\right)$ and $\sigma\left(\operatorname{Var}_{\text {Lin }}^{\text {out }}(G)\right) \cdot \operatorname{In}\left(G^{\prime}\right)$ are both linear.

\section{Example 10.}

Let Prog $=\{P(\widehat{g(x)}, y, z) \leftarrow Q(\widehat{x}, f(y), z)\}$ and $\left.G=P\left(g \widehat{\left(f\left(x^{\prime}\right)\right.}\right), y^{\prime}, z^{\prime}\right), R\left(\widehat{z^{\prime}}\right)$. Then $G \leadsto{ }_{\sigma} G^{\prime}$ with $\sigma=\left(x / f\left(x^{\prime}\right), y / y^{\prime}, z / z^{\prime}\right), G^{\prime}=Q\left(\widehat{f\left(x^{\prime}\right)}, f\left(y^{\prime}\right), z^{\prime}\right), R\left(\widehat{z^{\prime}}\right)$. Note that $G^{\prime}$ is loop free, $\sigma\left(\operatorname{Var}_{\text {Lin }}^{\text {in }}(G)\right)$.Out $\left(G^{\prime}\right)=\left(y^{\prime}, f\left(x^{\prime}\right), z^{\prime}\right)$ is linear, $\sigma\left(\operatorname{Var}_{\text {Lin }}^{\text {out }}(G)\right) \cdot \operatorname{In}\left(G^{\prime}\right)=\left(x^{\prime}, f\left(y^{\prime}\right), z^{\prime}\right)$ is linear.

Proof. First, we show that $\sigma\left(\operatorname{Var}_{\text {Lin }}^{\text {in }}(G)\right)$. Out $\left(G^{\prime}\right)$ and $\sigma\left(\operatorname{Var}_{\text {Lin }}^{\text {out }}(G)\right) \cdot \operatorname{In}\left(G^{\prime}\right)$ are linear. Thus, in a second time, we show that $G^{\prime}$ is loop free.

Suppose that $G \sim_{\sigma} G^{\prime}$. Thus, there exist an atom $A_{x}$ in $G=A_{1}, \ldots$, $A_{x}, \ldots, A_{n}$, an S-CF-clause $H \leftarrow B \in \operatorname{Prog}$ and the mgu $\sigma$ such that $\sigma(H)=$ $\sigma\left(A_{x}\right)$ and $G^{\prime}=\sigma(G)\left[\sigma\left(A_{x}\right) \leftarrow \sigma(B)\right]$.

Let $\operatorname{Var}_{L i n}^{i n}(G)=x_{1}, \ldots, x_{k}, \ldots, x_{k+n^{\prime}}, \ldots, x_{m}$ built as follows:

- $x_{1}, \ldots, x_{k-1}$ are the variables occurring in $\operatorname{Var}^{i n}\left(A_{1}, \ldots, A_{x-1}\right)$ and not in $\operatorname{Var}^{\text {out }}(G)$;

\footnotetext{
${ }^{7}$ If it is not the case then variables are relabelled.
} 
- $x_{k}, \ldots, x_{k+n^{\prime}}$ are the variables occurring in $\operatorname{Var}^{i n}\left(A_{x}\right)$ and not in $\operatorname{Var}^{\text {out }}(G)$;

- $x_{k+n^{\prime}+1}, \ldots, x_{m}$ are variables occurring the atoms $\operatorname{Var}^{i n}\left(A_{x+1}, \ldots, A_{n}\right)$ and not in $\operatorname{Var}^{\text {out }}(G)$.

Since $\operatorname{In}(G)$ and $\operatorname{Out}(G)$ are both linear and $\sigma$ is the mgu of $A_{x}$ and $H$, one has $\sigma\left(\operatorname{Var}_{\text {Lin }}^{i n}(G)\right)=x_{1}, \ldots, x_{k+1}, \sigma\left(x_{k}\right), \ldots, \sigma\left(x_{k+n^{\prime}}\right), x_{k+n^{\prime}+1}, \ldots x_{m}$. Note that the linearity of $\operatorname{In}(G)$ involves the linearity of $\operatorname{Var}_{L i n}^{i n}(G)$. And one can deduce that $\sigma\left(\operatorname{Var}_{L i n}^{i n}(G)\right)$ is linear iff the tuple $\sigma\left(x_{k}\right), \ldots, \sigma\left(x_{k+n^{\prime}}\right)$ is linear.

By hypothesis, $\operatorname{Out}(H) \cdot \operatorname{In}(B)$ and $\operatorname{Out}(B) \cdot \operatorname{In}(H)$ are both linear.

So, a variable occurring in $\operatorname{Var}(H) \cap \operatorname{Var}(B)$ is either

- a variable that is in $\operatorname{Out}(H)$ and $\operatorname{Out}(B)$ or

- a variable that is in $\operatorname{In}(H)$ and $\operatorname{In}(B)$.

A variable occurring in $\operatorname{Out}(H)$ and in $\operatorname{In}(H)$ does not occur in $B$. Symmetrically, a variable occurring in $\operatorname{Out}(B)$ and in $\operatorname{In}(B)$ does not occur in $H$. Moreover, a variable cannot occur twice in either $\operatorname{Out}(H)$ or $\operatorname{In}(H)$.

Let us focus on $A_{x} . A_{x}$ is linear since it does not contain any loop by hypothesis. Let us study the possible forms of $H$ given in Fig. 1.

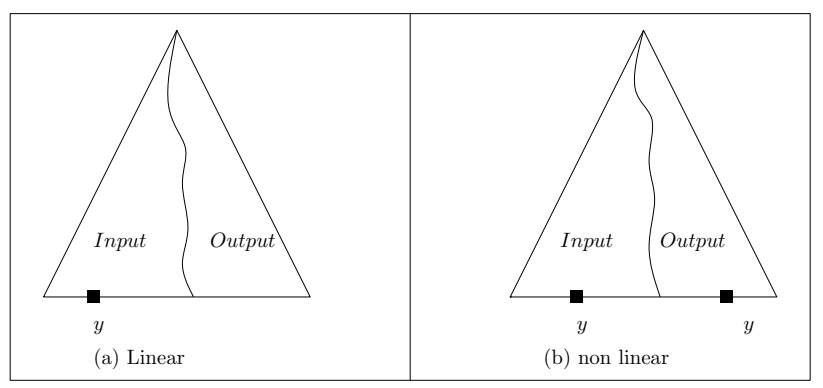

Figure 1: Possible forms of $H$

Each variable $y$ occurring in $B$ is:

- either a new variable or 
- a variable occurring once in $H$ and preserving its nature (input or output).

The relation $\sim_{\text {Prog }}$ ensures the nature stability of variables i.e.

$$
\begin{array}{r}
\operatorname{Var}(\operatorname{Out}(\sigma(B))) \cap \operatorname{Var}(\operatorname{In}(\sigma(H)))=\emptyset \text { and } \\
\operatorname{Var}(\operatorname{In}(\sigma(B))) \cap \operatorname{Var}(\operatorname{Out}(\sigma(H)))=\emptyset
\end{array}
$$

Moreover, a consequence of Lemma 1 is that $\operatorname{Out}(\sigma(B))$ and $\operatorname{In}(\sigma(B))$ are both linear.

Let us study the two possible cases:

(a) since the variables of $H$ and those of $G$ are distinct and $\operatorname{Var}_{\text {Lin }}^{i n}(G)$ is linear, $\sigma\left(\operatorname{Var}_{\text {Lin }}^{i n}(G)\right)=x_{1}, \ldots, x_{k+1}, \sigma\left(x_{k}\right), \ldots, \sigma\left(x_{k+n^{\prime}}\right), x_{k+n^{\prime}+1}, \ldots, x_{m}$ is also linear. Moreover, considering $H$ as linear and (1) and (2), a consequence is that

$$
\bigcup_{x_{i}, i \in\left\{k, \ldots, k+n^{\prime}\right\}} \operatorname{Var}\left(\sigma\left(x_{i}\right)\right) \subseteq\left\{x_{k}, \ldots, x_{k+n^{\prime}}\right\} \cup \operatorname{Var}^{i n}\left(A_{x}\right) .
$$

One can also deduce that $\operatorname{Var}^{\text {out }}\left(G^{\prime}\right) \subseteq \operatorname{Var}^{\text {out }}(G) \cup\left(\operatorname{Var}^{\text {out }}(B)\right)$. Consequently, $\operatorname{Var}^{\text {out }}\left(G^{\prime}\right) \cap \operatorname{Var}\left(\sigma\left(\operatorname{Var}_{\text {Lin }}^{\text {in }}(G)\right)\right)=\emptyset$ and the tuple $\sigma\left(\operatorname{Var}_{\text {Lin }}^{\text {in }}(\right.$ $G))$.Out $\left(G^{\prime}\right)$ is linear iff $\operatorname{Out}\left(G^{\prime}\right)$ is linear.

(b) A variable can occur at most twice in $H$ but an occurrence of such a variable is necessarily an input variable and the other an output variable. Consequently the unification between $A_{x}$ and $H$ leads to a variable $\alpha$ of $\sigma\left(\operatorname{Var}_{\operatorname{Lin}}^{i n}(G)\right)$ occurring twice in $\sigma(H)$. But according to the form of $H$, these two occurrences of $\alpha$ do not occur in $\sigma\left(\operatorname{Var}_{\text {Lin }}^{i n}(G)\right)$ since one of the two occurrences is necessarily at an output position. So $\sigma\left(\operatorname{Var}_{\text {Lin }}^{i n}(G)\right)=x_{1}, \ldots, x_{k+1}, \sigma\left(x_{k}\right), \ldots, \sigma\left(x_{k+n^{\prime}}\right), x_{k+n^{\prime}+1}, \ldots, x_{m}$ is a linear tuple. Moreover, Prog being a non-copying S-CF program, for any variable $x_{i}$, with $i=k, \ldots k+n^{\prime}$,

- if $x_{i} \in \operatorname{Var}(\sigma(x))$ with $x$ a variable occurring twice in $H$ then $\operatorname{Var}\left(\sigma\left(x_{i}\right)\right) \cap \operatorname{Var}^{\text {out }}\left(G^{\prime}\right)=\emptyset ;$ 
- if there exists $z \in \operatorname{Var}^{\text {out }}\left(A_{x}\right)$ s.t. $z \in \operatorname{Var}\left(\sigma\left(x_{i}\right)\right)$ and $z \in \operatorname{Var}(\sigma(x))$ with $x$ occurring twice in $H$ then $\operatorname{Var}\left(\sigma\left(x_{i}\right)\right) \cap \operatorname{Var}^{\text {out }}\left(G^{\prime}\right)=\emptyset$;

- if there exists $z \in \operatorname{Var}^{\text {out }}\left(A_{x}\right)$ s.t. $x_{i} \in \operatorname{Var}(\sigma(z))$ and $z \in \operatorname{Var}(\sigma(x))$ with $x$ occurring twice in $H$ then $\operatorname{Var}\left(\sigma\left(x_{i}\right)\right) \cap \operatorname{Var}^{\text {out }}\left(G^{\prime}\right)=\emptyset$;

- if there exists $x \in \operatorname{Var}^{i n}(H)$ such that $x \notin \operatorname{Var}^{\text {out }}(H)$ then one has $\operatorname{Var}\left(\sigma\left(x_{i}\right)\right) \subseteq\left\{x_{k}, \ldots, x_{k+n^{\prime}}\right\} \cup \operatorname{Var}^{i n}\left(A_{x}\right)$. Thus $\operatorname{Var}\left(\sigma\left(x_{i}\right)\right) \cap$ $\operatorname{Var}^{\text {out }}\left(G^{\prime}\right)=\emptyset$.

Consequently, $\sigma\left(\operatorname{Var}_{\text {Lin }}^{i n}(G)\right)$.Out $\left(G^{\prime}\right)$ is linear iff $\operatorname{Out}\left(G^{\prime}\right)$ is linear.

Let us now study the linearity of $\operatorname{Out}\left(G^{\prime}\right)$. First, let us focus on the case $\operatorname{Out}\left(\sigma\left(G-A_{x}\right)\right)$ where $G-A_{x}$ is the sequence of atoms $G$ for which the atom $A_{x}$ has been removed. Note that $\sigma\left(G-A_{x}\right)=G^{\prime}-\sigma(B)$.

Suppose that $\operatorname{Out}\left(G-A_{x}\right)$ is not linear. So there exist two distinct variables $x$ and $y$ of $G$ such that $\operatorname{Var}(\sigma(x)) \cap \operatorname{Var}(\sigma(y)) \neq \emptyset$. Since these variables are concerned by the mgu $\sigma$, they are also variables of $A_{x}$ at input positions as illustrated in Fig. 2. Since these variables are distinct and share the same variable by the application of $\sigma$, then there exist two subterms (red and green triangles in Fig. 2) at input positions in $H$ sharing the same variable $\alpha$. That is impossible since, by definition, for each $H \leftarrow B \in \operatorname{Prog}$, one has $\operatorname{In}(H)$.Out(B) and $\operatorname{Out}(H) \cdot \operatorname{In}(B)$ both linear.

So, the last possible case for breaking the linearity of $\operatorname{Out}\left(G^{\prime}\right)$ is that there exist two distinct variables $x$ and $y$ such that $x$ occurs in $O u t(B), y$ occurs in $\operatorname{Out}\left(G-A_{x}\right)$ and $\operatorname{Var}(\sigma(x)) \cap \operatorname{Var}(\sigma(y)) \neq \emptyset$. A variable $\alpha$ of $\operatorname{Var}(\sigma(x)) \cap$ $\operatorname{Var}(\sigma(y))$ is necessarily a variable of $H$. Since a copy of $\alpha$ is done in the variable $y$ and $y$ necessarily occurs in $A_{x}$ at an input position, there is a contradiction. Indeed, it means that the variable $\alpha$ must occur both in $\operatorname{Out}(H)$ and $\operatorname{In}(H)$ but also in $\operatorname{Out}(B)$. Thus, $H \leftarrow B$ is not a non-copying S-CF clause. Consequently, $\operatorname{Out}\left(G^{\prime}\right)$ is linear.

To conclude, $\sigma\left(\operatorname{Var}_{\operatorname{Lin}}^{i n}(G)\right)$.Out $\left(G^{\prime}\right)$ is linear. Note that showing that $\sigma($ $\left.\operatorname{Var}_{\text {Lin }}^{\text {out }}(G)\right) \cdot \operatorname{In}\left(G^{\prime}\right)$ is linear is similar. 


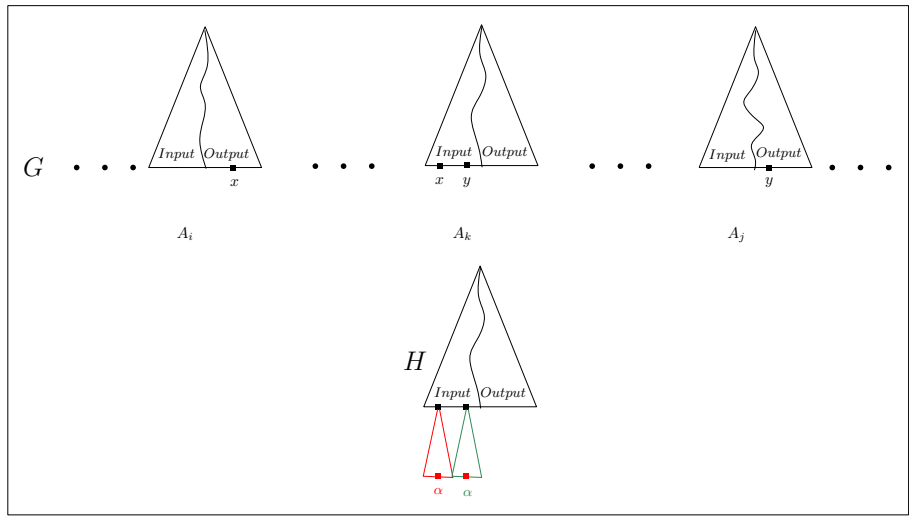

Figure 2: $G-A_{x}$

The last remaining point to show is that $G^{\prime}$ does not contain any loops.

By construction, $G^{\prime}=\sigma(G)\left[\sigma\left(A_{x}\right) \leftarrow \sigma(B)\right]$. There are three cases to study:

- Suppose there exists a loop occurring in $G^{\prime}-\sigma(B)$. By definition, $G^{\prime}-$ $\sigma(B)=\sigma\left(A_{1}\right), \ldots \sigma\left(A_{x-1}\right), \sigma\left(A_{x+1}\right), \ldots \sigma\left(A_{m}\right)$. Let us reason on the sequence of atoms $G$ where $G=A_{i}, A_{x}, A_{j}$. Note that it can be easily generalized to a sequence of atoms of any size, but for a matter of simplicity, we focus on a significant sequence composed of three atoms. In that case, $G^{\prime}-\sigma(B)=\sigma\left(A_{i}\right), \sigma\left(A_{j}\right)$. If there exists a loop in $G^{\prime}-\sigma(B)$ but not in $G$ then there are two possibilities (actually three but two of them are exactly symmetric):

- $A_{i} \nsucc A_{j}$ and $A_{j} \nsucc A_{i}$ : Then $\sigma$ has generated the loop. So, one can deduce that there exist two variables $\alpha$ and $\beta$ such that $\alpha \in$ $\operatorname{Var}^{\text {in }}\left(\sigma\left(A_{i}\right)\right) \cap \operatorname{Var}^{\text {out }}\left(\sigma\left(A_{j}\right)\right), \beta \in \operatorname{Var}^{\text {out }}\left(\sigma\left(A_{i}\right)\right) \cap \operatorname{Var}^{\text {in }}\left(\sigma\left(A_{j}\right)\right)$. Thus, there exist $y \in \operatorname{Var}^{\text {out }}\left(A_{i}\right), y^{\prime} \in \operatorname{Var}^{i n}\left(A_{i}\right), z \in \operatorname{Var}^{\text {out }}\left(A_{j}\right)$ and $z^{\prime} \in \operatorname{Var}^{i n}\left(A_{j}\right)$ such that $\alpha \in \operatorname{Var}\left(\sigma\left(y^{\prime}\right)\right) \cap \operatorname{Var}(\sigma(z))$ and $\beta \in$ $\operatorname{Var}(\sigma(y)) \cap \operatorname{Var}\left(\sigma\left(z^{\prime}\right)\right)$. Since those four variables are concerned by the mgu, one can deduce that they also occur in $A_{x}$. More precisely, according to the linearity of $\operatorname{In}(G)$ and $\operatorname{Out}(G), y^{\prime} \in \operatorname{Var}^{\text {out }}\left(A_{x}\right)$, $y \in \operatorname{Var}^{i n}\left(A_{x}\right), z \in \operatorname{Var}^{i n}\left(A_{x}\right)$ and $z^{\prime} \in \operatorname{Var}^{\text {out }}\left(A_{x}\right)$. In that case, 
$A_{i} \succ A_{x}$ and $A_{x} \succ A_{i}$ because $y^{\prime} \in \operatorname{Var}^{\text {out }}\left(A_{x}\right) \cap \operatorname{Var}^{i n}\left(A_{i}\right)$ and $y \in \operatorname{Var}^{\text {out }}\left(A_{i}\right) \cap \operatorname{Var}^{\text {in }}\left(A_{x}\right)$. Consequently, a loop occurs in $G$. Contradiction.

- $A_{i} \succ A_{j}$ and $A_{j} \nsucc A_{i}$ : Consequently, $\sigma$ has generated the loop. Since $A_{i} \succ A_{j}$, then there exists a variable $y$ such that $y \in \operatorname{Var}^{i n}\left(A_{i}\right) \cap$ $\operatorname{Var}^{\text {out }}\left(A_{j}\right)$. If there exists a loop in $G^{\prime}-\sigma(B)$ then there exists a variable $\alpha$ s.t. $\alpha \in \operatorname{Var}^{\text {out }}\left(\sigma\left(A_{i}\right)\right) \cap \operatorname{Var}^{i n}\left(\sigma\left(A_{j}\right)\right)$. So there exist two variables $y^{\prime}$ and $z^{\prime}$ with $y^{\prime} \in \operatorname{Var}^{\text {out }}\left(A_{i}\right)$ and $z^{\prime} \in \operatorname{Var}^{i n}\left(A_{j}\right)$ s.t. $\alpha \in$ $\operatorname{Var}\left(\sigma\left(y^{\prime}\right)\right) \cap \operatorname{Var}\left(\sigma\left(z^{\prime}\right)\right)$. Since those two variables are concerned by the mgu, one can deduce that they also occur in $A_{x}$. More precisely, according to the linearity of $\operatorname{In}(G)$ and $\operatorname{Out}(G), y^{\prime} \in \operatorname{Var}^{i n}\left(A_{x}\right)$ and $z^{\prime} \in \operatorname{Var}^{\text {out }}\left(A_{x}\right)$. In that case, one has $A_{x} \succ A_{i}$ and $A_{j} \succ A_{x}$ because $y^{\prime} \in \operatorname{Var}^{i n}\left(A_{x}\right) \cap \operatorname{Var}^{\text {out }}\left(A_{i}\right)$ and $z^{\prime} \in \operatorname{Var}^{\text {out }}\left(A_{x}\right) \cap \operatorname{Var}{ }^{i n}\left(A_{j}\right)$. Moreover, by hypothesis, $A_{i} \succ A_{j}$. Consequently, a loop occurs in $G$ because $A_{j} \succ A_{x} \succ A_{i} \succ A_{j}$. Contradiction.

- A loop cannot occur in $\sigma(B)$ : This is a direct consequence of Lemma 1. Indeed, $\sigma$ is the mgu of $A_{x}$ which is linear and $H . B$ is constructed from the variables occurring once in $H$ and new variables. Moreover, $\operatorname{In}(B)$ and $\operatorname{Out}(B)$ are linear and the only variables allowed to appear in both $\operatorname{In}(B)$ and $\operatorname{Out}(B)$ are necessarily new and then not instantiated by $\sigma$. To create a loop in these conditions would require that two different variables $\alpha$ and $\beta$ instantiated by $\sigma$ would share the same variable i.e. $\operatorname{Var}(\sigma(\alpha)) \cap \operatorname{Var}(\sigma(\beta)) \neq \emptyset$. Contradicting Lemma 1.

- Suppose that a loop occurs in $G^{\prime}$ but neither in $G^{\prime}-\sigma(B)$ nor in $\sigma(B)$ : Let $G$ be the sequence of atoms such that $G=A_{i}, A_{x}$. In that case, $G^{\prime}=\sigma\left(A_{i}\right), \sigma(B)$ with $\sigma$ the mgu of $A_{x}$ and $H$. One can extend the schema to any kind of sequence of atoms satisfying the hypothesis of this lemma without loss of generality. We consider $B$ as follows: $B=B_{1}, \ldots, B_{k}$. If there exists a loop in $G^{\prime}$ but neither in $G^{\prime}-\sigma(B)$ nor in $\sigma(B)$ then there exist $B_{k_{1}}, \ldots, B_{k_{n}}$ atoms occurring in $B$ such that $\sigma\left(A_{i}\right) \succ \sigma\left(B_{k_{1}}\right) \succ \ldots \succ$ 
$\sigma\left(B_{k_{n}}\right) \succ \sigma\left(A_{i}\right)$. So, one can deduce that there exists two variables $\alpha$ and $\beta$ such that $\alpha \in \operatorname{Var}^{\text {in }}\left(\sigma\left(A_{i}\right)\right) \cap \operatorname{Var}^{\text {out }}\left(\sigma\left(B_{k_{1}}\right)\right)$ and $\beta \in \operatorname{Var}^{\text {out }}\left(\sigma\left(A_{i}\right)\right) \cap$ $\left.\operatorname{Var}^{\text {out }}\left(\sigma\left(B_{k_{n}}\right)\right)\right)$. Consequently, there exists two variables $y, z$ such that $y \in \operatorname{Var}^{i n}\left(A_{i}\right), z \in \operatorname{Var}^{\text {out }}\left(A_{i}\right), \alpha \in \operatorname{Var}(\sigma(y))$ and $\beta \in \operatorname{Var}(\sigma(z))$. Both variables also occur in $A_{x}$. Suppose that $y$ does not occur in $A_{x}$. Since $\sigma$ is the mgu of $A_{x}$ and $H$ and $y$ not in $\operatorname{Var}\left(A_{x}\right), \sigma$ does not instantiate $y$. Consequently, $\alpha=y$. However, $\operatorname{Var}(\sigma(B)) \subseteq \operatorname{Var}(H) \cup \operatorname{Var}\left(A_{x}\right) \cup$ $\operatorname{Var}(B)$. Moreover, the sets of variables occurring in Prog and in $G$ are supposed to be disjointed. So, $y$ cannot occur in $\sigma(B)$ and then the loop in $G^{\prime}$ does not exist. Thus, $y$ occurs in $A_{x}$ as well as $z$. Furthermore, since $\operatorname{In}(G)$ and $\operatorname{Out}(G)$ are linear, $y \in \operatorname{Var}^{\text {out }}\left(A_{x}\right)$ and $z \in \operatorname{Var}^{i n}\left(A_{x}\right)$. Consequently, $G$ contains a loop. Contradicting the hypothesis.

To conclude, $G^{\prime}$ does not contain any loop.

Lemma 2 can be generalized to several steps.

Lemma 3. Let Prog be a non-copying S-CF program, and $G$ be a sequence of atoms such that $\operatorname{Out}(G)$ is linear, $\operatorname{In}(G)$ is linear and $G$ does not contain loops. We assume ${ }^{8}$ that variables occurring in Prog are different from those occurring in $G$. If $G \sim_{\sigma}^{*} G^{\prime}$, then $G^{\prime}$ is loop free, $\sigma\left(\operatorname{Var}_{L i n}^{\text {in }}(G)\right)$.Out $\left(G^{\prime}\right)$ and $\sigma\left(\operatorname{Var}_{\text {Lin }}^{\text {out }}(G)\right) \cdot \operatorname{In}\left(G^{\prime}\right)$ are both linear.

Proof. Let $G \sim_{\sigma}^{*} G^{\prime}$ be rewritten as follows: $G_{0} \sim_{\sigma_{1}} G_{1} \ldots \sim_{\sigma_{k}} G_{k}$ with $G_{0}=G, G^{\prime}=G_{k}$ and $\sigma=\sigma_{k} \circ \ldots \circ \sigma_{1}$. Let $P_{k}$ be the induction hypothesis defined such that: If $G_{0} \sim_{\sigma}^{*} G_{k}$ then

- $G_{k}$ does not contain any loop,

- $\sigma\left(\operatorname{Var}_{\text {Lin }}^{i n}\left(G_{0}\right)\right) . \operatorname{Out}\left(G_{k}\right)$ is linear and

- $\sigma\left(\operatorname{Var}_{\text {Lin }}^{\text {out }}\left(G_{0}\right)\right) \cdot \operatorname{In}\left(G_{k}\right)$ is linear.

\footnotetext{
${ }^{8}$ If it is not the case then variables are relabelled.
} 
Let us proceed by induction.

- $P_{0}$ is trivially true. Indeed, $\operatorname{In}\left(G_{0}\right)$ and $\operatorname{Out}\left(G_{0}\right)$ are linear. Moreover, for any $x \in \operatorname{Var}_{\text {Lin }}^{i n}\left(G_{0}\right)$ (resp. $\left.x \in \operatorname{Var}_{\text {Lin }}^{\text {out }}\left(G_{0}\right)\right)$, one has $x \notin \operatorname{Var}\left(\operatorname{Out}\left(G_{0}\right)\right)$ (resp. $x \notin \operatorname{Var}\left(\operatorname{In}\left(G_{0}\right)\right)$ ). Thus, $\operatorname{Var}_{\text {Lin }}^{\text {in }}\left(G_{0}\right)$.Out $\left(G_{0}\right)$ is linear (resp. $\left.\operatorname{Var}_{\text {Lin }}^{\text {out }}\left(G_{0}\right) \cdot \operatorname{In}\left(G_{0}\right)\right)$.

- Suppose that $P_{k}$ is true and $G_{k} \leadsto \sigma_{k+1} G_{k+1}$. Since $G_{k} \sim_{\sigma_{k+1}} G_{k+1}$, there exist $H \leftarrow B \in \operatorname{Prog}$ and an atom $A_{x}$ occurring in $G_{k}$ s.t. $\sigma_{k+1}$ is the mgu of $A_{x}$ and $H$, and $G_{k+1}=\sigma_{k+1}\left(G_{k}\right)\left[\sigma_{k+1}(H) \leftarrow \sigma_{k+1}(B)\right]$. By hypothesis, one has $\operatorname{Out}\left(G_{k}\right)$ and $\operatorname{In}\left(G_{k}\right)$ linear. Consequently, Lemma 2 can be applied and one obtains that

- $\sigma\left(\operatorname{Var}_{L i n}^{i n}\left(G_{k}\right)\right)$. Out $\left(G_{k+1}\right)$ is linear,

- $\sigma\left(\operatorname{Var}_{L i n}^{\text {out }}\left(G_{k}\right) \cdot \operatorname{In}\left(G_{k+1}\right)\right.$ is linear and

$-G_{k+1}$ does not contain any loop.

Moreover, for Prog a non-copying S-CF program, if $G_{i} \leadsto_{\sigma_{i+1}} G_{i+1}$ then one has: For any variable $x, y$, if $x \in \operatorname{Var}_{L i n}^{i n}\left(G_{i}\right)$ and $y \in \operatorname{Var}\left(\sigma_{i+1}(x)\right)$ then $y \in \operatorname{Var}_{L i n}^{i n}\left(G_{i+1}\right)$ or $y \notin \operatorname{Var}\left(G_{i+1}\right)$. So, one can conclude that given $\sigma_{k} \circ \ldots \circ \sigma_{1}\left(\operatorname{Var}_{L i n}^{i n}\left(G_{0}\right)\right)$, for any variable $x \in \operatorname{Var}_{L i n}^{i n}\left(G_{0}\right)$, for any $y \in \operatorname{Var}\left(\sigma_{k} \circ \ldots \circ \sigma_{1}(x)\right)$, either $y \in \operatorname{Var}_{L i n}^{i n}\left(G_{k}\right)$ or $y \notin \operatorname{Var}\left(G_{k}\right)$.

Let us study the variables of $\bigcup_{y \in \operatorname{Var}_{L i n}^{i n}\left(G_{0}\right)}\left(\operatorname{Var}\left(\sigma_{k} \circ \ldots \circ \sigma_{1}(y)\right)\right.$.

- For any variable $x$ s.t. $x \in \bigcup_{y \in \operatorname{Var}_{\text {Lin }}^{i n}\left(G_{0}\right)}\left(\operatorname{Var}\left(\sigma_{k} \circ \ldots \circ \sigma_{1}(y)\right)\right) \backslash$ $\operatorname{Var}\left(G_{k}\right), x \notin \operatorname{Var}\left(G_{k+1}\right)$. Indeed, an already-used variable cannot be reused for relabelling variables of Prog while the reduction process. Moreover such variables are not instantiated by $\sigma_{k+1}$ since the mgu $\sigma_{k+1}$ of $A_{x}$ and $H$ only concerns variables of $\operatorname{Var}(H) \cup \operatorname{Var}\left(A_{x}\right)$. So, for any variable $y$ in $\operatorname{Var}\left(\sigma_{k} \circ \ldots \circ \sigma_{1}(y)\right) \backslash \operatorname{Var}\left(G_{k}\right)$, one has $\sigma_{k+1}(y)=y$ and $y \notin \operatorname{Var}\left(G_{k+1}\right)$. Consequently, for any variable $y$ in $\left.\operatorname{Var}\left(\sigma_{k+1} \circ \sigma_{k} \circ \ldots \circ \sigma_{1}(y)\right)\right) \backslash \operatorname{Var}\left(G_{k}\right), y \notin \operatorname{Var}\left(G_{k+1}\right)$. 
- For any variable $x$ s.t. $x \in \bigcup_{y \in \operatorname{Var}_{L i n}^{i n}\left(G_{0}\right)}\left(\operatorname{Var}\left(\sigma_{k} \circ \ldots \circ \sigma_{1}(y)\right)\right) \cap$ $\operatorname{Var}\left(G_{k}\right)$, one can deduce that $x \in \operatorname{Var}_{L i n}^{i n}\left(G_{k}\right)$.

Since $\sigma_{k+1}\left(\operatorname{Var}_{L i n}^{i n}\left(G_{k}\right)\right)$. Out $\left(G_{k+1}\right)$ is linear, therefore one can deduce that for any $y \in \bigcup_{y \in \operatorname{Var}_{\operatorname{Lin}}^{i n}\left(G_{0}\right)}\left(\operatorname{Var}\left(\sigma_{k} \circ \ldots \circ \sigma_{1}(y)\right)\right) \cap \operatorname{Var}\left(G_{k}\right)$, $\operatorname{Var}\left(\sigma_{k+1} \circ \sigma_{k} \circ \ldots \circ \sigma_{1}(y)\right) \cap \operatorname{Var}\left(\operatorname{Out}\left(G_{k+1}\right)\right)=\emptyset$.

So, one has $\sigma_{k+1} \circ \sigma_{k} \circ \ldots \circ \sigma_{1}\left(\operatorname{Var}_{L i n}^{i n}\left(G_{k}\right)\right)$.Out $\left(G_{k+1}\right)$ is linear. The proof of $\sigma\left(\operatorname{Var}_{L i n}^{\text {out }}\left(G_{k}\right) \cdot \operatorname{In}\left(G_{k+1}\right)\right.$ is in some sense symmetric. To conclude, considering the hypothesis of Lemma 2, one has: If $G \sim_{\sigma}^{*} G^{\prime}$, then

$-G^{\prime}$ is loop free;

- $\sigma\left(\operatorname{Var}_{\text {Lin }}^{\text {in }}(G)\right)$. Out $\left(G^{\prime}\right)$ is linear;

- $\sigma\left(\operatorname{Var}_{L i n}^{\text {out }}(G)\right) \cdot \operatorname{In}\left(G^{\prime}\right)$ is linear.

\section{Computing Descendants}

Let us first present the main ideas.

Example 11. Let $R=\{f(x) \rightarrow g(h(x))\}$ and $I=\left\{p^{n}\left(f\left(s^{n}(a)\right)\right) \mid n \in \mathbb{I N}\right\}$ generated by Predicate $P_{0}$ in the $S$-CF program Prog $=$ $\left\{Q(\widehat{a}) \leftarrow . P_{0}(\widehat{x}) \leftarrow P_{1}(\widehat{x}, y), Q(\widehat{y}) . P_{1}(\widehat{p(x)}, y) \leftarrow P_{1}(\widehat{x}, s(y)) . P_{1}(\widehat{f(x)}, x) \leftarrow\right\}$. Note that $R^{*}(I)=I \cup\left\{p^{n}\left(g\left(h\left(s^{n}(a)\right)\right)\right) \mid n \in I N\right\}$.

To simulate the rewrite step $f\left(s^{n}(a)\right) \rightarrow g\left(h\left(s^{n}(a)\right)\right)$, we consider the rewriterule left-hand-side $f(x)$. We can see that:

$P_{1}(\widehat{f(x)}, y) \leadsto[\operatorname{Prog}, \theta=(x / y)] \emptyset$ and $\left.\theta\left(P_{1}(\widehat{f(x)}, y)\right)=P_{1}(\widehat{f(y)}, y) \rightarrow_{R} P_{1}(\widehat{g(h(y)}), y\right)$. Then the clause $\left.P_{1}(\widehat{g(h(y)}), y\right) \leftarrow$ is called critical pair ${ }^{9}$. This critical pair is not convergent (in Prog) because $\left.P_{1}(\widehat{g(h(y)}), y\right) \nrightarrow_{\text {Prog }}^{*} \emptyset$. To get the descendants, the critical pairs should be convergent. Let $\left.\operatorname{Prog}^{\prime}=\operatorname{Prog} \cup\left\{P_{1}(\widehat{g(h(y)}), y\right) \leftarrow\right\}$. Now the critical pair is convergent in Prog', and note that the predicate $P_{0}$ of

\footnotetext{
${ }^{9}$ In former work, a critical pair was a pair. Here it is a clause since we use logic programs.
} 
Prog generates $R^{*}(I)$. Adding critical pairs into the $S$-CF program is called completion.

For technical reasons ${ }^{10}$, we consider only normalized S-CF programs, and Prog' is not normalized. However, the critical pair can be normalized using a new predicate symbol, and replaced by the following normalized clauses $P_{1}(\widehat{g(x)}, y) \leftarrow P_{2}(\widehat{x}, y) . P_{2}(\widehat{h(y)}, y) \leftarrow$. This is the role of Function norm in the completion algorithm below.

In general, adding a critical pair (after normalizing it) into the S-CF program may create new critical pairs, and the completion process may not terminate. To force termination, two bounds predicate-limit and arity-limit are fixed. If predicate-limit is reached, Function norm should re-use existing predicates instead of creating new ones. If a new predicate symbol is created whose arity ${ }^{11}$ is greater than arity-limit, then this predicate has to be cut by Function norm into several predicates whose arities do not exceed arity-limit. On the other hand, for a fixed ${ }^{12}$ S-CF program, the number of critical pairs may be infinite. Function removeCycles modifies some clauses so that the number of critical pairs is finite. Strong coherence is technical and will be defined later. It is used to prove the results. However, if the initial program Prog is regular or is a CS-program, i.e. Prog does not have input arguments, then the strong coherence property is automatically satisfied.

Definition 9 (comp). Let arity-limit and predicate-limit be positive integers. Let $R$ be a linear rewrite system, and Prog be a finite, normalized and noncopying S-CF program strongly coherent with $R$. The completion process is defined by:

Function $\operatorname{comp}_{R}($ Prog $)$

Prog $=$ removeCycles $($ Prog $)$

while there exists a non-convergent critical pair $H \leftarrow B$ in Prog do

\footnotetext{
${ }^{10}$ Critical pairs are computed only at root positions.

${ }^{11}$ The number of arguments.

${ }^{12}$ i.e. without adding new clauses in the S-CF program.
} 


$$
\text { Prog }=\operatorname{removeCycles}\left(\operatorname{Prog} \cup \text { norm }_{\text {Prog }}(H \leftarrow B)\right)
$$

\section{end while}

return Prog

Critical pairs and strong coherence are defined in Section 4.1, and Theorem 2 shows closure under rewriting when all critical pairs are convergent. Theoretical notions and results are presented in Section 4.2 in order to define Function removeCycles. Section 4.3 speaks about normalization, and the final result, i.e. we get an over-approximation of the descendants, is given in Section 4.4.

\subsection{Critical pairs}

The notion of critical pair is the heart of our technique. Indeed, it allows us to add S-CF clauses into the current S-CF program in order to cover rewriting steps.

Definition 10. Let Prog be a non-copying S-CF program and $l \rightarrow r$ be a leftlinear rewrite rule. Consider distinct variables $x_{1}, \ldots, x_{n}$ such that $\operatorname{Var}(l) \cap$ $\left\{x_{1}, \ldots, x_{n}\right\}=\emptyset$. If there are $P$ and $k$ s.t. the $k^{\text {th }}$ argument of $P$ is an output, and $P\left(x_{1}, \ldots, x_{k-1}, l, x_{k+1}, \ldots, x_{n}\right) \sim_{\theta}^{+} G$ where $^{13}$

1. resolution steps are applied only on atoms whose output is not flat,

2. Out $(G)$ is flat and

3. the clause $P\left(t_{1}, \ldots, t_{n}\right) \leftarrow B$ used in the first step of this derivation satisfies $t_{k}$ is not a variable ${ }^{14}$

then the clause $\theta\left(P\left(x_{1}, \ldots, x_{k-1}, r, x_{k+1}, \ldots, x_{n}\right)\right) \leftarrow G$ is called critical pair. if $\theta$ does not instantiate the variables of $\operatorname{In}\left(P\left(x_{1}, \ldots, x_{k-1}, l, x_{k+1}, \ldots, x_{n}\right)\right)$ then the critical pair is said strict.

\footnotetext{
${ }^{13}$ Here, we do not use a hat to indicate output arguments because they may occur anywhere depending on $P$.

${ }^{14}$ In other words, the overlap of $l$ on the clause head $P\left(t_{1}, \ldots, t_{n}\right)$ is done at a non-variable position.
} 
Example 12. Let Prog be the $S-C F$ program defined by:

$\operatorname{Prog}=\{P(\widehat{s(x)}) \leftarrow Q(\widehat{x}, a) . \quad Q(\widehat{f(x)}, y) \leftarrow Q(\widehat{x}, g(y)) . \quad Q(\widehat{x}, x) \leftarrow$.$\} and con-$ sider $R=\{f(f(x)) \rightarrow h(x)\}$. Note that $L(P)=\left\{s\left(f^{n}\left(g^{n}(a)\right)\right) \mid n \in \mathbb{I N}\right\}$.

We have $Q(\widehat{f(f(x)}), y) \leadsto Q(\widehat{f(x)}, g(y)) \leadsto Q(\widehat{x}, g(g(y)))$.

Since Out $(Q(\widehat{x}, g(g(y))))$ is flat, this generates the strict critical pair $Q(\widehat{h(x)}, y) \leftarrow$ $Q(\widehat{x}, g(g(y)))$.

The following lemma is very important for completion. It shows that when the completion process adds a strict critical pair into the current S-CF program, the resulting program is still S-CF.

Lemma 4. A strict critical pair is an S-CF clause. In addition, if $l \rightarrow r$ is right-linear, a strict critical pair is a non-copying $S$-CF clause.

Proof. Let $G_{0}=P\left(x_{1}, \ldots, x_{k-1}, l, x_{k+1}, \ldots, x_{n}\right)$. Since $l$ is linear, $G_{0}$ is linear and $\operatorname{Var}_{\text {Lin }}^{i n}\left(G_{0}\right)=\operatorname{In}\left(G_{0}\right)$. From Lemma 3, $\theta\left(\operatorname{In}\left(G_{0}\right)\right)$.Out $(G)$ is linear and $G$ is loop-free. Note that $\operatorname{In}\left(G_{0}\right)$ and $\operatorname{Out}(G)$ are tuples of variables. Since the critical pair is strict,we deduce that $\theta$ does not instantiate the variables of $\operatorname{In}\left(G_{0}\right)$, then $\theta\left(\operatorname{In}\left(G_{0}\right)\right) \cdot \operatorname{Out}(G)$ is a linear tuple of variables. Consequently, a strict critical pair is an S-CF clause.

Since $G_{0}$ is linear, $\operatorname{Var}_{\text {Lin }}^{\text {out }}\left(G_{0}\right)=\operatorname{Var}^{\text {out }}\left(G_{0}\right)$. Thus, from Lemma 3, $\theta\left(\operatorname{Out}\left(G_{0}\right)\right) \cdot \operatorname{In}(G)$ is linear. And since $r$ is linear, the critical pair is a noncopying clause.

Definition 11. A critical pair $H \leftarrow B$ is said convergent if $H \rightarrow{ }_{\text {Prog }}^{*} B$.

The critical pair of Example 12 is not convergent.

Let us recall that the completion procedure is based on adding the nonconvergent critical pairs into the program. In order to preserve the nature of the S-CF program, the computed non-convergent critical pairs are expected to be strict. Moreover, since critical pairs are only computed using output arguments, each reducible ${ }^{15}$ symbol should not occur in an input argument. So

\footnotetext{
${ }^{15}$ I.e. the top symbol of the left-hand-side of some rewrite rule.
} 
we define a sufficient condition on $R$ and Prog called strong coherence.

Definition 12. Let $R$ be a rewrite system. We consider the smallest set of consuming symbols, recursively defined by: $f \in \Sigma$ is consuming if there exists a rewrite rule $f\left(t_{1}, \ldots, t_{n}\right) \rightarrow r$ in $R$ s.t. some $t_{i}$ is not a variable, or $r$ contains at least one consuming symbol.

The S-CF program Prog is strongly coherent with $R$ if:

1) for all $l \rightarrow r \in R$, the top-symbol of $l$ does not occur in input arguments of Prog,

2) and no consuming symbol occurs in clause-heads having input arguments.

Note that a CS-program (no input arguments) is strongly coherent with any rewrite system.

In $R=\{g(s(y)) \rightarrow h(y)\}, g$ is consuming. Thus $\operatorname{Prog}=\{P(\widehat{g(x)}, x) \leftarrow$. is not strongly coherent with $R$ because item 2 is not satisfied. We have $P(\widehat{g(s(y)}), z) \sim[x / s(y), z / s(y)] \emptyset$, which generates the critical pair $P(\widehat{h(y)}, s(y)) \leftarrow$. This critical pair is not a $\mathrm{S}-\mathrm{CF}$ clause.

Lemma 5. If Prog is a normalized $S$-CF program strongly coherent with $R$, then every critical pair cp is strict, and Prog $\cup\{c p\}$ is strongly coherent with $R$.

Proof. Consider $f(\vec{s}) \rightarrow r \in R$ ( $\vec{s}$ is a tuple of terms), and assume that

$$
P\left(\widehat{\overrightarrow{x_{1}}}, \widehat{f(\vec{s})}, \widehat{\overrightarrow{x_{2}}}, \vec{z}\right) \sim_{\left[P\left(\widehat{\overrightarrow{t_{1}}}, \widehat{f(\vec{u})}, \widehat{\overrightarrow{t_{2}}, \vec{v}} \leftarrow B, \theta\right]\right.} G \sim_{\sigma}^{*} G^{\prime}
$$

such that $\operatorname{Out}\left(G^{\prime}\right)$ is flat, $\overrightarrow{x_{1}}, \overrightarrow{x_{2}}, \vec{z}, \vec{u}, \vec{v}$ are tuples of distinct variables and $\overrightarrow{t_{1}}$, $\overrightarrow{t_{2}}$ are tuples of terms (however $\vec{v}$ may share some variables with $\overrightarrow{t_{1}} \cdot \overrightarrow{u_{1}} \cdot \overrightarrow{t_{2}}$ ). This derivation generates the critical pair $(\sigma \circ \theta)\left(P\left(\widehat{\overrightarrow{x_{1}}}, \widehat{r}, \widehat{\overrightarrow{x_{2}}}, \vec{z}\right)\right) \leftarrow G^{\prime}$.

If $l \rightarrow r$ is consuming then $P$ has no input arguments, i.e. $\vec{z}$ and $\vec{v}$ do not exist. Therefore $\sigma \circ \theta$ cannot instantiate the input variables of $P$, hence the critical pair is strict.

Otherwise $\vec{s}$ is a linear tuple of variables, and $(x / t$ means that the variable $x$ is replaced by $t) \theta=(\vec{v} / \vec{z}) \circ\left(\overrightarrow{x_{1}} / \overrightarrow{t_{1}}, \vec{s} / \vec{u}, \overrightarrow{x_{2}} / \overrightarrow{t_{2}}\right)$, which does not instantiate $\vec{z}$ nor the output variables of $B$. Moreover $\operatorname{Out}(B)$ is flat, then $\operatorname{Out}(G)=\operatorname{Out}(\theta B)$ 
is flat. Thus $G^{\prime}=G$ and the critical pair is $P\left(\widehat{\theta \overrightarrow{x_{1}}}, \widehat{\theta r}, \widehat{\theta \overrightarrow{x_{2}}}, \vec{z}\right) \leftarrow G$, which is strict.

About strong coherence, the function symbols occurring in the input arguments of the critical pair come from the input arguments of Prog. Therefore condition 1 of Definition 12 is satisfied.

On the other hand, suppose that $P$ has input arguments. So $f$ is not consuming, then $\vec{s}$ is a linear tuple of variables. Consequently the derivation contains only one step, i.e. $G^{\prime}=G$. Then $\vec{s}$ is instantiated by variables, and $\theta\left(\overrightarrow{x_{1}}\right)=\overrightarrow{t_{1}}$, $\theta\left(\overrightarrow{x_{2}}\right)=\overrightarrow{t_{2}}$, where $\overrightarrow{t_{1}}$ and $\overrightarrow{t_{2}}$ do not contain consuming symbols. Moreover $r$ does not contain consuming symbols (otherwise $f$ would be consuming). Therefore condition 2 of Definition 12 is satisfied.

So, we come to our main result that ensures to get the rewriting closure when every computable critical pair is convergent.

Theorem 2. Let $R$ be a linear rewrite system, and Prog be a non-copying normalized $S$-CF program strongly coherent with $R$. If all strict critical pairs are convergent, then for every predicate symbol $P$ without input arguments, $L(P)$ is closed under rewriting by $R$, i.e. $\left(\vec{t} \in L(P) \wedge \vec{t} \rightarrow_{R}^{*} \overrightarrow{t^{\prime}}\right) \Longrightarrow \overrightarrow{t^{\prime}} \in L(P)$.

The proof is very technical. To illustrate the proof, consider Example 12 again, except that the critical pair $Q(\widehat{h(x)}, y) \leftarrow Q\left(\widehat{x}, g^{2}(y)\right)$ is added into the S-CF program. Now

$$
\begin{aligned}
\operatorname{Prog}=\{ & P(\widehat{s(x)}) \leftarrow Q(\widehat{x}, a) . \quad Q(\widehat{f(x)}, y) \leftarrow Q(\widehat{x}, g(y)) . \quad Q(\widehat{x}, x) \leftarrow . \\
& \left.Q(\widehat{h(x)}, y) \leftarrow Q\left(\widehat{x}, g^{2}(y)\right) \quad\right\}
\end{aligned}
$$

and $R=\left\{f^{2}(x) \rightarrow h(x)\right\}$. So there is only one critical pair, which is convergent thanks to the last clause of Prog.

$s\left(f^{2}\left(g^{2}(a)\right)\right) \in L(P)$ because the atom $A=P\left(s\left(f^{2}\left(g^{2}(a)\right)\right)\right) \in \operatorname{Mod}(\operatorname{Prog})$, moreover $A=P\left(s\left(f^{2}\left(g^{2}(a)\right)\right)\right) \rightarrow_{R} A^{\prime}=P\left(s\left(h\left(g^{2}(a)\right)\right)\right)$. Since $A \in \operatorname{Mod}(\operatorname{Prog})$, we have $A \leadsto * \emptyset$.

More precisely: 
$\left.A=P\left(s\left(f^{2}\left(g^{2}(a)\right)\right)\right) \leadsto A^{\prime \prime}=Q\left(f^{2}\left(\widehat{g^{2}(a)}\right), a\right) \leadsto Q\left(f \widehat{\left(g^{2}(a)\right.}\right), g(a)\right) \leadsto$ $Q\left(\widehat{g^{2}(a)}, g^{2}(a)\right) \leadsto \emptyset$.

Using the notations of the proof, we have $C=s, l=f^{2}(x), r=h(x)$, $\sigma=\left(x / g^{2}(a)\right), \sigma^{\prime}=\left(x / g^{2}(a), y / a\right)$. Thus $A^{\prime \prime}=Q(\widehat{\sigma(l)}, a)=\sigma^{\prime}(Q(\widehat{l}, y))$.

On the other hand, since Prog is non-copying, we have

$$
\left.A^{\prime}=P\left(s\left(h\left(g^{2}(a)\right)\right)\right) \leadsto Q\left(\widehat{h\left(g^{2}(a)\right.}\right), a\right)=Q(\widehat{\sigma(r)}, a)=\sigma^{\prime}(Q(\widehat{h(x)}, y))
$$

where $Q(\widehat{h(x)}, y)$ is the head of the critical pair. Since the critical pair is convergent, we have

$$
A^{\prime} \leadsto \sigma^{\prime}(Q(\widehat{h(x)}, y)) \leadsto \sigma^{\prime}\left(Q\left(\widehat{x}, g^{2}(y)\right)\right)=Q\left(\widehat{g^{2}(a)}, g^{2}(a)\right) \leadsto \emptyset
$$

Therefore $A^{\prime} \in \operatorname{Mod}(\operatorname{Prog})$, hence $s\left(h\left(g^{2}(a)\right)\right) \in L(P)$.

Proof. (of Theorem 2) Let $A \in \operatorname{Mod}(\operatorname{Prog})$ s.t. $A \rightarrow_{l \rightarrow r} A^{\prime}$. Then $\left.A\right|_{i}=C[\sigma(l)]$ for some $i \in \mathbb{N}$ and $A^{\prime}=A[i \leftarrow C[\sigma(r)]$.

Since resolution is complete, $A \sim^{*} \emptyset$. Since Prog is normalized, resolution consumes symbols of $C$ one by one. Since Prog is coherent with $R$, the top symbol of $l$ cannot be generated as an input: it is either consumed in an output argument, or the whole $\sigma(l)$ disappears thanks to an output argument. Consequently $G_{0}=A \sim^{*} G_{k} \sim^{*} \emptyset$ and there exists an atom $A^{\prime \prime}=P\left(t_{1}, \ldots, t_{n}\right)$ in $G_{k}$ and an output argument $j$ s.t. $t_{j}=\sigma(l)$, i.e. $A \sim^{*} G_{k}\left[A^{\prime \prime}=P\left(t_{1}, \ldots, \sigma(l), \ldots, t_{n}\right)\right]$, and along the step $G_{k} \leadsto G_{k+1}$ the top symbol of $\sigma(l)$ is consumed or $\sigma(l)$ disappears entirely. On the other hand, $A^{\prime} \sim^{*} G_{k}\left[A^{\prime \prime} \leftarrow P\left(t_{1}, \ldots, \sigma(r), \ldots, t_{n}\right)\right]$ since Prog is non-copying.

If $t_{j}=\sigma(l)$ disappears entirely, it can be replaced by any term, then $A^{\prime} \sim^{*} G_{k}\left[A^{\prime \prime} \leftarrow P\left(t_{1}, \ldots, \sigma(r), \ldots, t_{n}\right)\right] \sim^{*} \emptyset$, hence $A^{\prime} \in \operatorname{Mod}(\operatorname{Prog})$. Otherwise the top symbol of $\sigma(l)$ is consumed along $G_{k} \leadsto G_{k+1}$. Consider new variables $x_{1}, \ldots, x_{n}$ such that $\left\{x_{1}, \ldots, x_{n}\right\} \cap \operatorname{Var}(l)=\emptyset$, and let us define the substitution $\sigma^{\prime}$ by $\forall i \in\{1, \ldots, n\}, \sigma^{\prime}\left(x_{i}\right)=t_{i}$ and $\forall x \in \operatorname{Var}(l), \sigma^{\prime}(x)=\sigma(x)$. Then $\sigma^{\prime}\left(P\left(x_{1}, \ldots, x_{j-1}, l, x_{j+1}, \ldots, x_{n}\right)\right)=A^{\prime \prime}$, and according to resolution properties $P\left(x_{1}, \ldots, l, \ldots, x_{n}\right) \sim_{\theta}^{*} \emptyset$ and $\theta \leq \sigma^{\prime}$. This derivation can be decomposed into: 
$P\left(x_{1}, \ldots, l, \ldots, x_{n}\right) \sim_{\theta_{1}}^{*} G^{\prime} \leadsto \theta_{\theta_{2}} G \sim_{\theta_{3}}^{*} \emptyset$ where $\theta=\theta_{3} \circ \theta_{2} \circ \theta_{1}$, and s.t. $\operatorname{Out}\left(G^{\prime}\right)$ is not flat and $\operatorname{Out}(G)$ is flat ${ }^{16}$.

The derivation $P\left(x_{1}, \ldots, l, \ldots, x_{n}\right) \sim_{\theta_{1}}^{*} G^{\prime} \sim_{\theta_{2}} G$ can be commuted into: $P\left(x_{1}, \ldots, l, \ldots, x_{n}\right) \sim_{\gamma_{1}}^{*} B^{\prime} \leadsto \gamma_{2} B \sim_{\gamma_{3}}^{*} G$ s.t. $\operatorname{Out}(B)$ is flat, $\operatorname{Out}\left(B^{\prime}\right)$ is not flat, and within $P\left(x_{1}, \ldots, l, \ldots, x_{n}\right) \sim_{\gamma_{1}}^{*} B^{\prime} \leadsto \gamma_{2} B$ resolution is applied only on atoms whose output is not flat, and we have $\gamma_{3} \circ \gamma_{2} \circ \gamma_{1}=\theta_{2} \circ \theta_{1}$. Then $\gamma_{2} \circ$ $\gamma_{1}\left(P\left(x_{1}, \ldots, r, \ldots, x_{n}\right)\right) \leftarrow B$ is a critical pair. By hypothesis, it is convergent, then $\gamma_{2} \circ \gamma_{1}\left(P\left(x_{1}, \ldots, r, \ldots, x_{n}\right)\right) \rightarrow^{*} B$. Note that $\gamma_{3}(B) \rightarrow^{*} G$ and recall that $\theta_{3} \circ \gamma_{3} \circ \gamma_{2} \circ \gamma_{1}=\theta_{3} \circ \theta_{2} \circ \theta_{1}=\theta$. Then $\theta\left(P\left(x_{1}, \ldots, r, \ldots, x_{n}\right)\right) \rightarrow^{*} \theta_{3}(G) \rightarrow^{*} \emptyset$, and since $\theta \leq \sigma^{\prime}$ we get $P\left(t_{1}, \ldots, \sigma(r), \ldots, t_{n}\right)=\sigma^{\prime}\left(P\left(x_{1}, \ldots, r, \ldots, x_{n}\right)\right) \rightarrow^{*} \emptyset$. Thus $A^{\prime} \sim^{*} G_{k}\left[A^{\prime \prime} \leftarrow P\left(t_{1}, \ldots, \sigma(r), \ldots, t_{n}\right)\right] \sim^{*} \emptyset$, hence $A^{\prime} \in \operatorname{Mod}(\operatorname{Prog})$.

By trivial induction, the proof can be extended to the case of several rewrite steps.

\subsection{Ensuring finitely many critical pairs}

The following example illustrates a situation where the number of critical pairs is infinite for a given S-CF program.

Example 13. Let $f(c(x), y) \rightarrow d(y)$ be a rewrite rule, and $\left\{P_{0}(\widehat{f(x, y)}) \leftarrow\right.$ $P_{1}(\widehat{x}, \widehat{y}) . \quad P_{1}(\widehat{x}, \widehat{s(y)}) \leftarrow P_{1}(\widehat{x}, \widehat{y}) . \quad P_{1}(\widehat{c(x)}, \widehat{y}) \leftarrow P_{2}(\widehat{x}, \widehat{y}) . P_{2}(\widehat{a}, \widehat{a}) \leftarrow$. $\}$ be an $S$-CF program ${ }^{17}$ Then $P_{0}(\widehat{f(\widehat{c(x)}, y)}) \rightarrow P_{1}(\widehat{c(x)}, \widehat{y}) \sim y / s(y) P_{1}(\widehat{c(x)}, \widehat{y}) \sim y / s(y)$ $\cdots P_{1}(\widehat{c(x)}, \widehat{y}) \rightarrow P_{2}(\widehat{x}, \widehat{y})$. Resolution is applied only on non-flat atoms and the last atom obtained by this derivation is flat. The composition of substitutions along this derivation gives $y / s^{n}(y)$ for some $n \in \mathbb{I}$. There are infinitely many such derivations, which generates infinitely many critical pairs of the form $\left.P_{0}\left(\widehat{\left(s^{n}(y)\right.}\right)\right) \leftarrow P_{2}(\widehat{x}, \widehat{y})$.

This is annoying since the completion process introduced in Definition 9

\footnotetext{
${ }^{16}$ Since $\emptyset$ is flat, a goal having a flat output can always be reached, i.e. in some cases $G=\emptyset$.

${ }^{17}$ Note that $L\left(P_{0}\right)=\left\{f\left(c(a), s^{n}(a)\right) \mid n \in \mathbb{N}\right\}$ is a regular language, whereas the S-CF program (which is also a CS-program) is not regular. If it were regular, there would be finitely many critical pairs.
} 
needs to compute all critical pairs. This is why we define sufficient conditions to ensure that a given finite S-CF program has finitely many critical pairs.

Definition 13. Prog is empty-recursive if there exist a predicate symbol $P$ and two tuples $\vec{x}=\left(x_{1}, \ldots, x_{n}\right), \vec{y}=\left(y_{1}, \ldots, y_{k}\right)$ composed of distinct variables s.t. $P(\widehat{\vec{x}} \cdot \vec{y}) \leadsto_{\sigma}^{+} A_{1}, \ldots, P\left(\widehat{\overrightarrow{x^{\prime}} \cdot \overrightarrow{t^{\prime}}}\right), \ldots, A_{k}$ where $\overrightarrow{x^{\prime}}=\left(x_{1}^{\prime}, \ldots, x_{n}^{\prime}\right)$ is a tuple of variables and there exist $i, j$ s.t. $x_{i}^{\prime}=\sigma\left(x_{i}\right)$ and $\sigma\left(x_{j}\right)$ is not a variable and $x_{j}^{\prime} \in \operatorname{Var}\left(\sigma\left(x_{j}\right)\right)$.

Example 14. Let Prog be the $S-C F$ program defined as follows: Prog = $\left\{P(\widehat{a}, \widehat{b}) \leftarrow . P\left(\widehat{x^{\prime}}, \widehat{s\left(y^{\prime}\right)}\right) \leftarrow P\left(\widehat{x^{\prime}}, \widehat{y^{\prime}}\right) \cdot\right\}$. From $P(\widehat{x}, \widehat{y})$, one can obtain the following derivation: $P(\widehat{x}, \widehat{y}) \leadsto\left[x / x^{\prime}, y / s\left(y^{\prime}\right)\right] P\left(\widehat{x^{\prime}}, \widehat{y^{\prime}}\right)$. Consequently, Prog is empty-recursive since $\sigma=\left[x / x^{\prime}, y / s\left(y^{\prime}\right)\right], x^{\prime}=\sigma(x)$ and $y^{\prime}$ is a variable of $\sigma(y)=s\left(y^{\prime}\right)$.

The following lemma shows that the non empty-recursiveness of an S-CF program is sufficient to ensure the finiteness of the number of critical pairs.

Lemma 6. Let Prog be a normalized S-CF program. If Prog is not emptyrecursive, then the number of critical pairs is finite.

Remark: Note that the S-CF program of Example 13 is normalized and has infinitely many critical pairs.

However it is empty-recursive because $P_{1}(\widehat{x}, \widehat{y}) \leadsto\left[x / x^{\prime}, y / s\left(y^{\prime}\right)\right] P_{1}\left(\widehat{x^{\prime}}, \widehat{y^{\prime}}\right)$.

Proof. By contrapositive. Let us suppose there exist infinitely many critical pairs. So there exist $P_{1}$ and infinitely many derivations of the form (i): $P_{1}\left(x_{1}, \ldots, x_{k-1}, l, x_{k+1}, \ldots, x_{n}\right) \sim_{\alpha}^{*} G^{\prime} \sim_{\theta} G$ (the number of steps is not bounded). As the number of predicates is finite and every predicate has a fixed arity, there exists a predicate $P_{2}$ and a derivation of the form

(ii): $P_{2}\left(t_{1}, \ldots, t_{p}\right) \sim_{\sigma}^{k} G_{1}^{\prime \prime}, P_{2}\left(t_{1}^{\prime}, \ldots, t_{p}^{\prime}\right), G_{2}^{\prime \prime}$ (with $k>0$ ) included in some derivation of $(i)$, strictly before the last step, such that:

1. $\operatorname{Out}\left(G_{1}^{\prime \prime}\right)$ and $\operatorname{Out}\left(G_{2}^{\prime \prime}\right)$ are flat and the derivation from $P_{2}\left(t_{1}, \ldots, t_{p}\right)$ can be applied on $P_{2}\left(t_{1}^{\prime}, \ldots, t_{p}^{\prime}\right)$ again, which gives rise to an infinite derivation. 
2. $\sigma$ is not empty and there exists a variable $x$ in $P_{2}\left(t_{1}, \ldots, t_{p}\right)$ such that $\sigma(x)=t$ and $t$ is not a variable and contains a variable $y$ that occurs in $P_{2}\left(t_{1}^{\prime}, \ldots, t_{p}^{\prime}\right)$. Otherwise $\sigma \circ \ldots \circ \sigma$ would always be a variable renaming and there would be finitely many critical pairs.

3. There is at least one non-variable term (let $t_{j}$ ) in output arguments of $P_{2}\left(t_{1}, \ldots, t_{p}\right)$ (due to the definition of critical pairs) such that $t_{j}^{\prime}=t_{j}{ }^{18}$. As we use an S-CF clause in each derivation step, the output argument $t_{j}^{\prime}$ matches a variable (output argument) in the body of the last clause used in $(i i)$. As $t_{j}^{\prime}=t_{j}$, the output argument $t_{j}$ matches a variable (output argument) in head of the first clause used in $(i i)$. So, for each variable $x$ occurring in the non-variable output terms of $P_{2}$, we have $\sigma(x)=x$.

4. From the previous item, we deduce that the variable $x$ found in item 2 is one of the terms $t_{1}, \ldots, t_{p}$, say $t_{k}$. We can assume that $y$ is $t_{k}^{\prime} \cdot t_{k}$ is an output argument of $P_{2}$ because it matches a non-variable and only output arguments are non-variable in the head of S-CF clause.

If in derivation $(i i)$ we replace all non-variable output terms by new variables, we obtain a new derivation ${ }^{19}$

(iii): $P_{2}\left(x_{1}, \ldots, x_{n}, t_{n+1}, \ldots, t_{p}\right) \sim_{\sigma^{\prime}}^{k} G_{1}^{\prime \prime \prime}, P_{2}\left(x_{1}^{\prime}, \ldots, x_{n}^{\prime}, t_{n+1}^{\prime}, \ldots, t_{p}^{\prime}\right), G_{2}^{\prime \prime \prime}$ and there exists $i, k$ (in $\{1, \ldots n\}$ ) such that $\sigma^{\prime}\left(x_{i}\right)=x_{i}^{\prime}$ (at least one non-variable term (in output arguments) in the (ii) derivation), and $\sigma^{\prime}\left(x_{k}\right)=t_{k}, x_{k}^{\prime}$ is a variable of $t_{k}$. We conclude that Prog is empty-recursive.

Deciding the empty-recursiveness of an S-CF program seems to be a difficult problem (undecidable ?). Nevertheless, we propose a sufficient syntactic condition to ensure that an S-CF program is not empty-recursive.

\footnotetext{
${ }^{18}$ This property does not necessarily hold as soon as $P_{2}$ is reached within (ii). We may have to consider further occurrences of $P_{2}$ so that each required term occurs in the required argument, which will necessarily happen because there are only finitely many permutations.

${ }^{19}$ Without loss of generality, we can consider that the output arguments (at least two) are the first arguments of $P_{2}$.
} 


\section{Definition 14.}

The $S$-CF clause $P\left(\widehat{t_{1}}, \ldots, \widehat{t_{n}}, x_{1}, \ldots, x_{k}\right) \leftarrow A_{1}, \ldots, Q(\ldots), \ldots, A_{m}$ is pseudoempty over $Q$ if there exist $i, j$ s.t.

- $t_{i}$ is a variable,

- and $t_{j}$ is not a variable,

- and $\exists x \in \operatorname{Var}\left(t_{j}\right), x \neq t_{i} \wedge\left\{x, t_{i}\right\} \subseteq \operatorname{VarOut}(Q(\ldots))$.

Roughly speaking, when making a resolution step issued from the following flat atom $P\left(\widehat{y_{1}}, \ldots, \widehat{y_{n}}, z_{1}, \ldots, z_{k}\right)$, the variable $y_{i}$ is not instantiated, and $y_{j}$ is instantiated by something that is synchronized with $y_{i}$ (in $Q(\ldots)$ ).

The $S$-CF clause $H \leftarrow B$ is pseudo-empty if there exists some $Q$ s.t. $H \leftarrow B$ is pseudo-empty over $Q$. The $S$-CF clause:

$P\left(\widehat{t_{1}}, \ldots, \widehat{t_{n}}, x_{1}, \ldots, x_{n^{\prime}}\right) \leftarrow A_{1}, \ldots, Q\left(\widehat{y_{1}}, \ldots, \widehat{y_{k}}, s_{1}, \ldots, s_{k^{\prime}}\right), \ldots, A_{m}$ is empty over $Q$ if for all $y_{i},\left(\exists j, t_{j}=y_{i}\right.$ or $\left.y_{i} \notin \operatorname{Var}\left(P\left(\widehat{t_{1}}, \ldots, \widehat{t_{n}}, x_{1}, \ldots, x_{n^{\prime}}\right)\right)\right)$.

Example 15. The $S$-CF clause $P(\widehat{x}, \widehat{f(x)}, \widehat{z}) \leftarrow Q(\widehat{x}, \widehat{z})$ is both pseudo-empty (thanks to the second and the third argument of $P$ ) and empty over $Q$ (thanks to the first and the third argument of $P$ ).

Definition 15. Using Definition 14, let us define two relations over predicate symbols.

- $P_{1} \unrhd_{\text {Prog }} P_{2}$ if there exists in Prog a clause empty over $P_{2}$ of the form $P_{1}(\ldots) \leftarrow A_{1}, \ldots, P_{2}(\ldots), \ldots, A_{n}$. The reflexive-transitive closure of $\unrhd_{\text {Prog }}$ is denoted by $\unrhd_{\text {Prog }}^{*}$.

- $P_{1}>_{\text {Prog }} P_{2}$ if there exist in Prog predicates $P_{1}^{\prime}$, $P_{2}^{\prime}$ s.t. $P_{1} \unrhd_{\text {Prog }}^{*} P_{1}^{\prime}$ and $P_{2}^{\prime} \unrhd_{\text {Prog }}^{*} P_{2}$, and a clause pseudo-empty over $P_{2}^{\prime}$ of the form $P_{1}^{\prime}(\ldots) \leftarrow A_{1}, \ldots, P_{2}^{\prime}(\ldots), \ldots, A_{n}$. The transitive closure of $>_{\text {Prog }}$ is denoted by $>_{\text {Prog }}^{+}$.

Prog is cyclic if there exists a predicate P s.t. $P>_{\text {Prog }}^{+} P$. 
Example 16. Let $\Sigma=\left\{f^{\backslash 1}, h^{\backslash 1}, a^{\backslash 0}\right\}$. Let Prog be the $S$-CF program such that $\operatorname{Prog}=\{P(\widehat{x}, \widehat{h(y)}, \widehat{f(z)}) \leftarrow Q(\widehat{x}, \widehat{z}), R(\widehat{y}) . Q(\widehat{x}, \widehat{g(y, z)}) \leftarrow P(\widehat{x}, \widehat{y}, \widehat{z}) . \quad Q(\widehat{a}, \widehat{a}) \leftarrow$. $R(\widehat{a}) \leftarrow$.$\} . One has P>_{\text {Prog }} Q$ and $Q>_{\text {Prog }} P$. Thus, Prog is cyclic.

The lack of cycles is the key point of our technique since it ensures the finiteness of the number of critical pairs.

Lemma 7. If Prog is not cyclic, then Prog is not empty-recursive, consequently the number of critical pairs is finite.

Proof. By contrapositive. Suppose that Prog is empty recursive. It exists $P$ s.t. $P\left(\widehat{x_{1}}, \ldots, \widehat{x_{n}}, y_{1}, \ldots, y_{n^{\prime}}\right) \leadsto A_{\sigma}^{+}, \ldots, P\left(\widehat{x_{1}^{\prime}}, \ldots, \widehat{x_{n}^{\prime}}, t_{1}^{\prime}, \ldots, t_{n^{\prime}}^{\prime}\right), \ldots, A_{k}$ where $x_{1}^{\prime}, \ldots, x_{n}^{\prime}$ are variables and there exist $i, j$ s.t. $x_{i}^{\prime}=\sigma\left(x_{i}\right)$ and $\sigma\left(x_{j}\right)$ is not a variable and $x_{j}^{\prime} \in \operatorname{Var}\left(\sigma\left(x_{j}\right)\right)$. We can extract from the previous derivation the following derivation which has $p$ steps $(p \geq 1)$ :

$P\left(\widehat{x_{1}}, \ldots, \widehat{x_{n}}, y_{1}, \ldots, y_{n^{\prime}}\right)=Q^{0}\left(\widehat{x_{1}}, \ldots, \widehat{x_{n}}, y_{1}, \ldots, y_{n^{\prime}}\right) \leadsto \alpha_{1}$ $B_{1}^{1} \ldots Q^{1}\left(\widehat{x_{1}^{1}}, \ldots, \widehat{x_{n_{1}}^{1}}, t_{1}^{1}, \ldots, t_{n_{1}^{\prime}}^{1}\right) \ldots B_{k_{1}}^{1} \leadsto \alpha_{2}$ $\left.B_{1}^{1} \ldots B_{1}^{2} \ldots Q^{2} \widehat{\left(x_{1}^{2}\right.}, \ldots, \widehat{x_{n_{2}}^{2}}, t_{1}^{2}, \ldots, t_{n_{2}^{\prime}}^{2}\right) \ldots B_{k_{2}}^{2} \ldots B_{k_{1}}^{1} \sim_{\alpha_{3}} \ldots \leadsto \alpha_{p}$ $B_{1}^{1} \ldots B_{1}^{p} \ldots Q^{p}\left(\widehat{x_{1}^{p}}, \ldots, \widehat{x_{n_{p}}^{p}}, t_{1}^{p}, \ldots, t_{n_{p}^{\prime}}^{p}\right) \ldots B_{k_{p}}^{p} \ldots B_{k_{1}}^{1}$ where $Q^{p}\left(\widehat{x_{1}^{p}}, \ldots, \widehat{x_{n_{p}}^{p}}, t_{1}^{p}, \ldots, t_{n_{p}^{\prime}}^{p}\right)=P\left(\widehat{x_{1}^{\prime}}, \ldots, \widehat{x_{n}^{\prime}}, t_{1}^{\prime}, \ldots, t_{n^{\prime}}^{\prime}\right)$.

For each $k$ (after $k$ steps in the previous derivation), $\alpha_{k} \circ \alpha_{k-1} \ldots \circ \alpha_{1}\left(x_{i}\right)$ is a variable of $\operatorname{Out}\left(Q^{k}\left(\widehat{x_{1}^{k}}, \ldots, \widehat{x_{n_{k}}^{k}}, t_{1}^{k}, \ldots, t_{n_{k}^{\prime}}^{k}\right)\right)$ and $\alpha_{k} \circ \alpha_{k-1} \ldots \circ \alpha_{1}\left(x_{j}\right)$ is either a variable of $\operatorname{Out}\left(Q^{k}\left(\widehat{x_{1}^{k}}, \ldots, \widehat{x_{n_{k}}^{k}}, t_{1}^{k}, \ldots, t_{n_{k}^{\prime}}^{k}\right)\right)$ or a non-variable term containing a variable of $\operatorname{Out}\left(Q^{k}\left(\widehat{x_{1}^{k}}, \ldots, \widehat{x_{n_{k}}^{k}}, t_{1}^{k}, \ldots, t_{n_{k}^{\prime}}^{k}\right)\right)$.

Each derivation step issued from $Q^{k}$ uses either a clause pseudo-empty over $Q^{k+1}$ and we deduce $Q^{k}>_{\text {Prog }} Q^{k+1}$, or an empty clause over $Q^{k+1}$ and we deduce $Q^{k} \unrhd_{\text {Prog }} Q^{k+1}$. At least one step uses a pseudo-empty clause otherwise no variable from $x_{1}, \ldots, x_{n}$ would be instantiated by a non-variable term containing at least one variable in $x_{1}^{\prime}, \ldots, x_{n}^{\prime}$.

We conclude that $P=Q^{0} o p_{1} Q^{1} o p_{2} Q^{2} \ldots Q^{p-1} o p_{p} Q^{p}=P$ with each $o p_{i}$ is $>_{\text {Prog }}$ or $\unrhd_{\text {Prog }}$ and there exists $k$ such that $o p_{k}$ is $>_{\text {Prog }}$. Therefore $P>_{\text {Prog }}^{+} P$, so Prog is cyclic. 
Thus, if Prog is not cyclic, all is fine. Otherwise, we need to transform Prog into $\operatorname{Prog}^{\prime}$ such as $\operatorname{Prog}^{\prime}$ is not cyclic and $\operatorname{Mod}(\operatorname{Prog}) \subseteq \operatorname{Mod}\left(\operatorname{Prog}^{\prime}\right)$.

The transformation is based on the following observation. If Prog is cyclic, there is at least one pseudo-empty clause that participates in a cycle. In Example 16, $P(\widehat{x}, \widehat{h(y)}, \widehat{f(z)}) \leftarrow Q(\widehat{x}, \widehat{z}), R(\widehat{y})$ is a pseudo-empty clause over $Q$ involved in the cycle. To remove the cycle, we transform it into $P(\widehat{x}, \widehat{h(y)}, \widehat{f(z)}) \leftarrow$ $Q\left(\widehat{x}, \widehat{x_{2}}\right), R\left(\widehat{x_{1}}\right), Q\left(\widehat{x_{3}}, \widehat{z}\right), R(\widehat{y})\left(x_{1}, x_{2}, x_{3}\right.$ are new variables), which is not pseudoempty anymore. The main process is described in Definition 19. Definitions 16, 17 and 18 are preliminary definitions used in Definition 19. Example 17 illustrates the definitions. If there are input arguments then some variables occurring in the input arguments of the body should also be renamed in order to get a non-copying S-CF clause.

Definition 16. Given a $S$-CF program Prog, the set $S$ of productive predicate symbols is recursively defined as being the smallest set s.t.

- for each fact $(P(\cdots) \leftarrow)$ of Prog, $P \in S$,

- and for each clause $P(\cdots) \leftarrow P_{1}(\cdots), \ldots, P_{n}(\cdots)$ of Prog, if $P_{1}, \ldots, P_{n} \in$ $S$, then $P \in S$.

$P$ is unproductive iff $P \notin S$.

Definition 17 (simplify). Let $H \leftarrow A_{1}, \ldots, A_{n}$ be an $S$-CF clause, and for each $i$, let us write $A_{i}=P_{i}(\ldots)$.

If there exists $P_{i}$ such that $P_{i}$ is unproductive then $\operatorname{simplify}\left(H \leftarrow A_{1}, \ldots, A_{n}\right)$ is the empty set, otherwise it is the set that contains only the S-CF clause $H \leftarrow B_{1}, \ldots, B_{m}$ such that

- $\left\{B_{i} \mid 0 \leq i \leq m\right\} \subseteq\left\{A_{i} \mid 0 \leq i \leq n\right\}$ and

- $\forall i \in\{1, \ldots, n\}, \quad\left(\neg\left(\exists j, B_{j}=A_{i}\right) \Leftrightarrow\left(\operatorname{Var}\left(A_{i}\right) \cap \operatorname{Var}(H)=\emptyset \wedge\right.\right.$ $\left.\left.\forall k \neq i, \operatorname{Var}\left(A_{i}\right) \cap \operatorname{Var}\left(A_{k}\right)=\emptyset\right)\right)$.

In other words, simplify deletes unproductive clauses, or it removes the atoms of the body that contain only free variables. 
Let $H \leftarrow B$ be a non-copying S-CF clause. Note that if the variable $x$ occurs several times in $B$ then $x \notin \operatorname{Var}(H)$.

Definition 18 (unSync). Let $H \leftarrow B$ be a non-copying $S$-CF clause.

Let us write $\operatorname{Out}(H)=\left(t_{1}, \ldots, t_{n}\right)$ and $\operatorname{In}(B)=\left(s_{1}, \ldots, s_{k}\right)$.

unSync $(H \leftarrow B)=\operatorname{simplify}\left(H \leftarrow \sigma_{0}(B), \sigma_{1}(B)\right)$ where $\sigma_{0}$, $\sigma_{1}$ are substitutions built as follows. $\forall x \in \operatorname{Var}(B)$ :

$\sigma_{0}(x)=\left\{\begin{array}{l}x \text { if } x \in \operatorname{VarOut}(B) \wedge \exists i, t_{i}=x \\ x \text { if } x \in \operatorname{VarIn}(B) \cap \operatorname{Var} I n(H) \wedge \exists j,\left(s_{j}=x\right) \\ \text { a fresh variable otherwise }\end{array}\right.$

$\sigma_{1}(x)=\left\{\begin{array}{l}x \text { if } x \in \operatorname{VarOut}(B) \wedge \exists i,\left(t_{i} \notin \operatorname{Var} \wedge x \in \operatorname{Var}\left(t_{i}\right)\right) \\ x \text { if } x \in \operatorname{VarIn}(B) \cap \operatorname{Var} I n(H) \wedge \exists j,\left(s_{j} \notin \operatorname{Var} \wedge x \in \operatorname{Var}\left(s_{j}\right)\right) \\ \text { a fresh variable otherwise }\end{array}\right.$

Definition 19 (removeCycles). Let Prog be an $S$-CF program. If Prog is not cyclic then removeCycles $(\operatorname{Prog})=\operatorname{Prog}$. Otherwise removeCycles $(\operatorname{Prog})=$ removeCycles $\left(\{\operatorname{unSync}(H \leftarrow B)\} \cup \operatorname{Prog}^{\prime}\right)$ where $H \leftarrow B$ is a pseudo-empty clause involved in a cycle and Prog' $=$ Prog $\backslash\{H \leftarrow B\}$.

Example 17. Let Prog be the $S$-CF program of Example 16. Since Prog is cyclic, let us compute removeCycles(Prog). The pseudo-empty S-CF clause $P(\widehat{x}, \widehat{h(y)}, \widehat{f(z)}) \leftarrow Q(\widehat{x}, \widehat{z}), R(\widehat{y})$ is involved in the cycle. Consequently, unSync is applied on it. According to Definition 18, one obtains $\sigma_{0}$ and $\sigma_{1}$ where $\sigma_{0}=\left[x / x, y / x_{1}, z / x_{2}\right]$ and $\sigma_{1}=\left[x / x_{3}, y / y, z / z\right]$. Thus, one gets the $S-C F$ clause $P(\widehat{x}, \widehat{h(y)}, \widehat{f(z)}) \leftarrow Q\left(\widehat{x}, \widehat{x_{2}}\right), R\left(\widehat{x_{1}}\right), Q\left(\widehat{x_{3}}, \widehat{z}\right), R(\widehat{y})$. Note that according to Definition 18, simplify is applied and removes $R\left(\widehat{x_{1}}\right)$ from the body. Following Definitions 17 and $19, P(\widehat{x}, \widehat{h(y)}, \widehat{f(z)}) \leftarrow Q(\widehat{x}, \widehat{z}), R(\widehat{y})$ is removed from Prog and $P(\widehat{x}, \widehat{h(y)}, \widehat{f(z)}) \leftarrow Q\left(\widehat{x}, \widehat{x_{2}}\right), Q\left(\widehat{x_{3}}, \widehat{z}\right), R(\widehat{y})$ is added instead. Note that the atom $R\left(\widehat{x_{1}}\right)$ has been removed using simplify. Note also that there is no cycle anymore.

removeCycles may enlarge the least Herbrand Model. 
Lemma 8. Let Prog and Prog' be two S-CF programs such that Prog is noncopying and Prog $^{\prime}=\operatorname{removeCycles}(\operatorname{Prog})$. Then Prog ${ }^{\prime}$ is a non-copying and non-cyclic S-CF program, and $\operatorname{Mod}(\operatorname{Prog}) \subseteq \operatorname{Mod}\left(\operatorname{Prog}^{\prime}\right)$. Moreover:

- if Prog is normalized, then so is Prog',

- if Prog is strongly coherent with R, then so is Prog'.

Consequently, there are finitely many critical pairs in $\operatorname{Prog}^{\prime}$.

Proof. removeCycles applies unSync until the program is not cyclic. When applying unSync, one pseudo-empty clause is removed and replaced by a nonpseudo-empty one. Thus, the number of pseudo-empty clauses decreases, and when there are no more pseudo-empty clauses, the program is not cyclic. Then removeCycles terminates and returns Prog $^{\prime}$, which is not cyclic.

simplify does not change $\operatorname{Mod}(\operatorname{Prog})$. On the other hand, unSync may enlarge $\operatorname{Mod}(\operatorname{Prog})$, because of the introduction of free variables in the clause body.

simplify and unSync do not change the clause head. Then if the clause is normalized, the resulting clause also is. Moreover, the fresh variables introduced into input arguments by $\sigma_{0}$ are distinct from those introduced by $\sigma_{1}$. Then if the clause is non-copying, the resulting clause also is.

The definition of strong coherence (Definition 12) includes two conditions. Recall that for a S-CF clause, function symbols in input arguments necessarily occur in the body. Therefore condition 1 is preserved by removeCycles because removeCycles does not add new function symbols in clause bodies (just new variables are added). Condition 2 is preserved by removeCycles because removeCycles does not change clause heads.

\subsection{Normalizing critical pairs - norm Prog}

If a critical pair is not convergent, we add it into Prog, and the critical pair becomes convergent. However, a critical pair is not necessarily normalized, whereas all clauses in Prog should be normalized. In the case of CS-clauses (i.e. without input arguments), a procedure that transforms a non-normalized clause

into normalized ones has been presented [2]. For example, $P(\widehat{f(g(x)}), \widehat{b}) \leftarrow Q(\widehat{x})$ 
can be normalized into $\left\{P(\widehat{f(x)}, \widehat{b}) \leftarrow P_{1}(\widehat{x}) . \quad P_{1}(\widehat{g(x)}) \leftarrow Q(\widehat{x}).\right\}\left(P_{1}\right.$ is a new predicate symbol). Since only output arguments should be normalized, this procedure still works even if there are also input arguments.

As new predicate symbols are introduced, possibly with bigger arities, completion may not terminate. To make it terminate in every case, two positive integers are used: predicate-limit and arity-limit. If the number of predicate symbols having the same arity as $P_{1}$ (including $P_{1}$ ) exceeds predicate-limit, an existing predicate symbol (for example $Q$ ) must be used instead of the new predicate $P_{1}$. This may enlarge $\operatorname{Mod}(\operatorname{Prog})$ in general and may lead to a strict over-approximation. If the arity of $P_{1}$ exceeds arity-limit, $P_{1}$ must be replaced in the clause body by several predicate symbols ${ }^{20}$ whose arities are less than or equal to arity-limit. This may also enlarge Mod(Prog). See [2] for more details. In other words norm $\operatorname{Prog}(H \leftarrow B)$ builds a set of normalized S-CF clauses $N$ such that $\operatorname{Mod}(\operatorname{Prog} \cup\{H \leftarrow B\}) \subseteq \operatorname{Mod}(\operatorname{Prog} \cup N)$.

However, when starting from a CS-program (i.e. without input arguments), it could be interesting to normalize by introducing input arguments, in order to profit from the bigger expressiveness of S-CF programs, and consequently to get a better approximation of the set of descendants, or even an exact computation, like in Examples 18 and 19 presented in Section 5. The quality of the approximation depends on the way the normalization is achieved. Some heuristics will be developed in further work. Moreover, they should preserve strong coherence when introducing new input arguments. A rule to preserve it could be as follows. For each function symbol $f$ occurring in the head of a critical pair:

- if $f$ is consuming, $f$ should be generated as output in a predicate symbol having no input arguments,

\footnotetext{
${ }^{20}$ For instance, if $P_{1}$ is binary and arity-limit $=1$, then $P_{1}\left(t_{1}, t_{2}\right)$ should be replaced by the sequence of atoms $P_{2}\left(t_{1}\right), P_{3}\left(t_{2}\right)$. Note that the dependency between $t_{1}$ and $t_{2}$ is lost, which may enlarge $\operatorname{Mod}(\operatorname{Prog})$. Symbols $P_{2}$ and $P_{3}$ are new if it is compatible with predicate-limit. Otherwise former predicate symbols should be used instead of $P_{2}$ and $P_{3}$.
} 
- if $f$ is reducible, i.e. $f$ occurs as the root of a left-hand-side, and $f$ is not consuming, $f$ should be generated as output.

This rule is applied in Examples 18 and 19.

\subsection{Completion}

At the beginning of Section 4, we have presented in Definition 9 the completion algorithm i.e. $\operatorname{comp}_{R}$. In Sections 4.1 and 4.3, we have described how to detect non-convergent critical pairs and how to convert them into normalized clauses using norm Prog $_{\text {. }}$

Theorem 3 illustrates that our technique leads to a finite S-CF program whose language over-approximates the descendants obtained by a linear rewrite system $R$.

Theorem 3. Function comp always terminates, and all critical pairs are convergent in $\operatorname{comp}_{R}($ Prog). Moreover, for each predicate symbol $P$ without input arguments, $R^{*}\left(L_{\text {Prog }}(P)\right) \subseteq L_{\operatorname{comp}_{R}(\text { Prog })}(P)$.

Proof. The proof is straightforward.

\section{Examples}

In this section, completion is applied on several examples. $I$ is the initial set of terms and $R$ is the rewrite system. Initially, we define an S-CF program Prog that generates $I$ and that satisfies the assumptions of Definition 9. To make the procedure terminate shortly, we suppose that predicate-limit=1, which means that for all $i$, there is at most one predicate symbol having $i$ arguments, except for $i=1$ we allow two predicate symbols having one argument.

When the following example is dealt with synchronized languages, i.e. with CS-programs [2, Example 42], we get a strict over-approximation of the descendants. Now, thanks to the bigger expressivity of S-CF programs, we compute the descendants in an exact way. 
Example 18. Let $I=\{f(a, a)\}$ and $R=\{f(x, y) \rightarrow u(f(v(x), w(y)))\}$. The exact set of descendants is $R^{*}(I)=\left\{u^{n}\left(f\left(v^{n}(a), w^{n}(a)\right)\right) \mid n \in \mathbb{N}\right\}$. We define Prog $=\left\{P_{f}(\widehat{f(x, y)}) \leftarrow P_{a}(\widehat{x}), P_{a}(\widehat{y}) .(\mathbf{1}), P_{a}(\widehat{a}) \leftarrow\right.$. (2) $\}$. Note that $L_{\text {Prog }}\left(P_{f}\right)=I$.

Using clause (1) we have $P_{f}(\widehat{f(x, y)}) \rightarrow{ }_{(1)} P_{a}(\widehat{x}), P_{a}(\widehat{y})$ generating the critical pair: $P_{f}(u(f(\widehat{v(x), w}(y)))) \leftarrow P_{a}(\widehat{x}), P_{a}(\widehat{y})$. In order to normalize this critical pair, we choose to generate symbols $u, f$ as output, $v, w$ as input. Moreover only one predicate symbol of arity 3 is allowed. It produces three new $S$-CF clauses:

$P_{f}(\widehat{z}) \leftarrow P_{1}(\widehat{z}, x, y), P_{a}(\widehat{x}), P_{a}(\widehat{y}) .(3), P_{1}(\widehat{u(z)}, x, y) \leftarrow P_{1}(\widehat{z}, v(x), w(y))$. (4) and $P_{1}(\widehat{f(x, y)}, x, y) \leftarrow$. (5).

Now $\left.P_{f}\left(\widehat{f\left(x^{\prime}, y^{\prime}\right)}\right) \rightarrow{ }_{(3)} P_{1}\left(\widehat{f\left(x^{\prime}, y^{\prime}\right.}\right), x, y\right), P_{a}(\widehat{x}), P_{a}(\widehat{y}) \leadsto(5), \sigma P_{a}(\widehat{x}), P_{a}(\widehat{y})$ where $\sigma=\left(x^{\prime} / x, y^{\prime} / y\right)$. Consequently, it generates the convergent critical pair $P_{f}(u(f(\widehat{v(x), w}(y)))) \leftarrow P_{a}(\widehat{x}), P_{a}(\widehat{y})$ again. On the other hand, since $\left.P_{1}\left(\widehat{f\left(x^{\prime}, y^{\prime}\right.}\right), x, y\right) \leadsto(5),\left(x^{\prime} / x, y^{\prime} / y\right) \emptyset$, the critical pair $P_{1}(u(f(\widehat{v(x), w}(y))), x, y) \leftarrow$ is detected, but it is already convergent.

No other critical pair is detected. Then, we get the S-CF program Prog' composed of clauses (1) to (5), and note that $L_{\text {Prog' }}\left(P_{f}\right)=R^{*}(I)$ indeed.

The run of the completion is summarized in Fig 3. The left-most column reports the detected non-convergent critical pairs and the right-most column describes how they are normalized.

\begin{tabular}{|c|l|}
\hline Detected non-convergent critical pairs & New clauses obtained by norm Prog \\
\hline & Starting S-CF program \\
& $P_{f}(f(\widehat{x, y})) \leftarrow P_{a}(\widehat{x}), P_{a}(\widehat{y})$. \\
& $P_{a}(\widehat{a}) \leftarrow$. \\
\hline$P_{f}\left(u(f(v(\widehat{x)}, w(y)))) \leftarrow P_{a}(\widehat{x}), P_{a}(\widehat{y})\right.$. & $P_{f}(\widehat{z}) \leftarrow P_{1}(\widehat{z}, x, y), P_{a}(\widehat{x}), P_{a}(\widehat{y})$. \\
& $P_{1}(\widehat{u(z)}, x, y) \leftarrow P_{1}(\widehat{z}, v(x), w(y))$. \\
& $P_{1}(f(x, y), x, y) \leftarrow$. \\
\hline$\emptyset$ & \\
\hline
\end{tabular}

Figure 3: Run of $\operatorname{comp}_{R}$ on Example 18 
The previous example could probably be dealt in an exact way using the technique of [1] as well, since $R^{*}(I)$ is a context-free language. It is not the case for the following example, whose language of descendants $R^{*}(I)$ is not contextfree (and not synchronized). It can be handled by S-CF programs in an exact way thanks to their bigger expressivity.

Example 19. Let $I=\left\{d_{1}(a, a, a)\right\}$ and

$$
R=\left\{\begin{array}{ll}
d_{1}(x, y, z) \stackrel{1}{\rightarrow} d_{1}(h(x), i(y), s(z)), & d_{1}(x, y, z) \stackrel{2}{\rightarrow} d_{2}(x, y, z) \\
d_{2}(x, y, s(z)) \stackrel{3}{\rightarrow} d_{2}(f(x), g(y), z), & d_{2}(x, y, a) \stackrel{4}{\rightarrow} c(x, y)
\end{array}\right\}
$$

$R^{*}(I)$ is composed of all terms appearing in the following derivation:

$$
\begin{aligned}
d_{1}(a, a, a) \stackrel{1}{\rightarrow}{ }^{n} & d_{1}\left(h^{n}(a), i^{n}(a), s^{n}(a)\right) \stackrel{2}{\rightarrow} d_{2}\left(h^{n}(a), i^{n}(a), s^{n}(a)\right) \\
& \stackrel{3}{\rightarrow} d_{2}\left(f^{k}\left(h^{n}(a)\right), g^{k}\left(i^{n}(a)\right), s^{n-k}(a)\right) \stackrel{4}{\rightarrow} c\left(f^{n}\left(h^{n}(a)\right), g^{n}\left(i^{n}(a)\right)\right) .
\end{aligned}
$$

Note that the last rewrite step by rule 4 is possible only when $k=n$.

$$
\text { Let Prog } \left.=\left\{P_{d}\left(d_{1} \widehat{(x, y, z}\right)\right) \leftarrow P_{a}(\widehat{x}), P_{a}(\widehat{y}), P_{a}(\widehat{z}) .(\mathbf{1}), P_{a}(\widehat{a}) \leftarrow \text {. (2) }\right\} \text {. }
$$

Thus $L_{\text {Prog }}\left(P_{d}\right)=I$.

By applying clause (1) and using rule 1, we get the critical pair:

$P_{d}\left(d_{1}(h(\widehat{x), i(y)}, s(z))) \leftarrow P_{a}(\widehat{x}), P_{a}(\widehat{y}), P_{a}(\widehat{z})\right.$. To normalize it, we choose to generate all symbols as output. Then the following clauses (3) and (4) are added into Prog: $\left.P_{d}\left(d_{1} \widehat{(x, y, z}\right)\right) \leftarrow P_{1}(\widehat{x}, \widehat{y}, \widehat{z})$. (3) and $P_{1}(\widehat{h(x)}, \widehat{i(y)}, \widehat{s(z)}) \leftarrow$ $P_{a}(\widehat{x}), P_{a}(\widehat{y}), P_{a}(\widehat{z})$. (4). By applying clause (1) and using rule 2 , we obtain the critical pair $\left.P_{d}\left(d_{2} \widehat{(x, y, z}\right)\right) \leftarrow P_{a}(\widehat{x}), P_{a}(\widehat{y}), P_{a}(\widehat{z})$. (5). This critical pair being already normalized, it is directly added into Prog.

We obtain the critical pair $P_{d}\left(d_{1}(h(\widehat{x), i(y)}, s(z))) \leftarrow P_{1}(\widehat{x}, \widehat{y}, \widehat{z})\right.$ by applying clause (3) and rule 1. To normalize it, we generate all symbols as output. It produces clause (3) again, and $P_{1}(\widehat{h(x)}, \widehat{i(y)}, \widehat{s(z)}) \leftarrow P_{1}(\widehat{x}, \widehat{y}, \widehat{z})$. (6).

Applying clause (3) and using rule 2, we get the critical pair: $\left.P_{d}\left(d_{2} \widehat{(x, y, z}\right)\right) \leftarrow P_{1}(\widehat{x}, \widehat{y}, \widehat{z})$. (7) which is already normalized. Thus, it is directly added into Prog. Applying clause (5) and using rule 4, we get the critical pair $P_{d}(\widehat{c(x, y)}) \leftarrow P_{a}(\widehat{x}), P_{a}(\widehat{y})$. (8) which is already normalized. Consequently, it is directly added into Prog.

By applying clauses (7) and (4), and using rule 3, we get the critical pair: 
$P_{d}\left(d_{2}\left(f(h(\widehat{x)), g}(i(y)), z)) \leftarrow P_{a}(\widehat{x}), P_{a}(\widehat{y}), P_{a}(\widehat{z})\right.\right.$. To normalize it, we choose to generate $d_{2}, f, g$ as output, and $h, i$ as input. It produces:

$$
\begin{aligned}
& P_{d}\left(d_{2} \widehat{(x, y, z)}\right) \leftarrow P_{2}\left(\widehat{x}, \widehat{y}, \widehat{z}, x^{\prime}, y^{\prime}, z^{\prime}\right), P_{a}\left(\widehat{x^{\prime}}\right), P_{a}\left(\widehat{y^{\prime}}\right), P_{a}\left(\widehat{z^{\prime}}\right) . \quad(\mathbf{9}) \\
& P_{2}\left(\widehat{f(x)}, \widehat{g(y)}, \widehat{z}, x^{\prime}, y^{\prime}, z^{\prime}\right) \leftarrow P_{2}\left(\widehat{x}, \widehat{y}, \widehat{z}, h\left(x^{\prime}\right), i\left(y^{\prime}\right), z^{\prime}\right) \quad\left(\mathbf{1 0}^{\prime}\right) \\
& P_{2}(\widehat{x}, \widehat{y}, \widehat{z}, x, y, z) \leftarrow . \quad(\mathbf{1 1})
\end{aligned}
$$

Now, clause $\left(10^{\prime}\right)$ may provide an infinite number of critical pairs. Applying removeCycles makes clause $\left(10^{\prime}\right)$ be substituted by the clause $P_{2}(\widehat{f(x)}, \widehat{g(y)}$, $\left.\widehat{z}, x^{\prime}, y^{\prime}, z^{\prime}\right) \leftarrow P_{2}\left(\widehat{x}, \widehat{y}, \widehat{z_{1}}, h\left(x^{\prime}\right), i\left(y^{\prime}\right), z_{1}^{\prime}\right), P_{2}\left(\widehat{x_{1}}, \widehat{y_{1}}, \widehat{z}, h\left(x_{1}^{\prime}\right), i\left(y_{1}^{\prime}\right), z^{\prime}\right)(\mathbf{1 0})$.

By applying clauses (7) and (6), and using rule 3, we get the critical pair: $P_{d}\left(d_{2}\left(f(h(\widehat{x)), g}(i(y)), z)) \leftarrow P_{1}(\widehat{x}, \widehat{y}, \widehat{z})\right.\right.$. We normalize it as previously. We get $\left.P_{d}\left(d_{2} \widehat{(x, y, z}\right)\right) \leftarrow P_{2}\left(\widehat{x}, \widehat{y}, \widehat{z}, x^{\prime}, y^{\prime}, z^{\prime}\right), P_{1}\left(\widehat{x^{\prime}}, \widehat{y^{\prime}}, \widehat{z^{\prime}}\right)$. (12) as well as (10), (11) again.

With clauses (9 or 12), (10), and rule 3, we get the convergent critical pairs $P_{d}\left(d_{2}\left(f(f(\widehat{x)), g}(g(y)), z)) \leftarrow P_{2}\left(\widehat{x}, \widehat{y}, \widehat{z_{1}}, h\left(h\left(x^{\prime}\right)\right), i\left(i\left(y^{\prime}\right)\right), z_{1}^{\prime}\right), P_{a}\left(\widehat{x^{\prime}}\right), P_{a}\left(\widehat{y^{\prime}}\right), P_{a}(\widehat{z})\right.\right.$ and $P_{d}\left(d_{2}\left(f(f(\widehat{x)), g}(g(y)), z)) \leftarrow P_{2}\left(\widehat{x}, \widehat{y}, \widehat{z_{1}}, h\left(h\left(x^{\prime}\right)\right), i\left(i\left(y^{\prime}\right)\right), z_{1}^{\prime}\right), P_{1}\left(\widehat{x^{\prime}}, \widehat{y^{\prime}}, \widehat{z}\right)\right.\right.$.

By applying clauses (9 or 12) and (11), and using rule 3, we get the convergent critical pairs $P_{d}\left(d_{2}\left(f(h(\widehat{x)), g}(i(y)), z)) \leftarrow P_{a}(\widehat{x}), P_{a}(\widehat{y}), P_{a}(\widehat{z})\right.\right.$ and $P_{d}($ $d_{2}\left(f(h(\widehat{x)), g}(i(y)), z)) \leftarrow P_{1}(\widehat{x}, \widehat{y}, \widehat{z})\right.$. By applying clauses (9) and (11), and using rule 4 , we get the convergent critical pair $P_{d}(\widehat{c(x, y)}) \leftarrow P_{a}(\widehat{x}), P_{a}(\widehat{y})$. Applying clauses (9) and (10), and using rule 4, we obtain the critical pair: $P_{d}(c(f \widehat{(x), g}(y))) \leftarrow P_{2}\left(\widehat{x}, \widehat{y}, \widehat{z}, h\left(x^{\prime}\right), i\left(y^{\prime}\right), z^{\prime}\right), P_{a}\left(\widehat{x^{\prime}}\right), P_{a}\left(\widehat{y^{\prime}}\right)$. Its normalization gives the clauses: $P_{3}(\widehat{f(x)}, \widehat{g(y)}) \leftarrow P_{2}\left(\widehat{x}, \widehat{y}, \widehat{z}, h\left(x^{\prime}\right), i\left(y^{\prime}\right), z^{\prime}\right), P_{a}\left(\widehat{x^{\prime}}\right), P_{a}\left(\widehat{y^{\prime}}\right)$. (13) and $P_{d}(\widehat{c(x, y)}) \leftarrow P_{3}(\widehat{x}, \widehat{y})$. (14). Note that the symbols $c, f$ and $g$ have been considered as output parameters.

No more critical pairs are detected and the procedure stops. The resulting program Prog' is composed of clauses (1) to (14). Note that the subset of descendants $d_{2}\left(f^{k}\left(h^{n}(a)\right), g^{k}\left(i^{n}(a)\right), s^{n-k}(a)\right)$ can be seen (with $p=n-k$ ) as $d_{2}\left(f^{k}\left(h^{k+p}(a)\right), g^{k}\left(i^{k+p}(a)\right), s^{p}(a)\right)$. The reader can check by himself that $L_{\text {Prog }^{\prime}}\left(P_{d}\right)$ is exactly $R^{*}(I)$.

The run of the completion on this example is also summarized in Fig 4. 
Black arrows means that the non-convergent critical pair is directly added to Prog since it is already normalized.

\begin{tabular}{|c|c|}
\hline Detected non-convergent critical pairs & New clauses obtained by norm $P r o g$ \\
\hline & $\begin{array}{l}\text { Starting S-CF program } \\
P_{d}\left(d_{1}(\widehat{x, y}, z)\right) \leftarrow P_{a}(\widehat{x}), P_{a}(\widehat{y}), P_{a}(\widehat{z}) . \\
P_{a}(\widehat{a}) \leftarrow .\end{array}$ \\
\hline$P_{d}\left(d_{1}(h(x), \widehat{i}(y), s(z))\right) \leftarrow P_{a}(\widehat{x}), P_{a}(\widehat{y}), P_{a}(\widehat{z})$ & $\begin{array}{l}P_{d}\left(d_{1}(\widehat{x, y}, z)\right) \leftarrow P_{1}(\widehat{x}, \widehat{y}, \widehat{z}) \\
P_{1}(\widehat{h(x)}, \widehat{i(y)}, \widehat{s(z)}) \leftarrow P_{a}(\widehat{x}), P_{a}(\widehat{y}), P_{a}(\widehat{z})\end{array}$ \\
\hline$P_{d}\left(d_{2}(\widehat{x, y}, z)\right) \leftarrow P_{a}(\widehat{x}), P_{a}(\widehat{y}), P_{a}(\widehat{z})$ & $\rightarrow$ \\
\hline$\left.P_{d}\left(d_{1}(h(x), \widehat{i(y}), s(z)\right)\right) \leftarrow P_{1}(\widehat{x}, \widehat{y}, \widehat{z})$ & $P_{1}(\widehat{h(x)}, \widehat{i(y)}, \widehat{s(z)}) \leftarrow P_{1}(\widehat{x}, \widehat{y}, \widehat{z})$. \\
\hline$P_{d}\left(d_{2}(\widehat{x, y}, z)\right) \leftarrow P_{1}(\widehat{x}, \widehat{y}, \widehat{z})$. & $\rightarrow$ \\
\hline$P_{d}(\widehat{c(x, y)}) \leftarrow P_{a}(\widehat{x}), P_{a}(\widehat{y})$. & $\rightarrow$ \\
\hline$P_{d}\left(d_{2}(f(h(x)), g(i(y)), z)\right) \leftarrow P_{a}(\widehat{x}), P_{a}(\widehat{y}), P_{a}(\widehat{z})$ & $\begin{array}{l}P_{d}\left(d_{2}(\widehat{x, y}, z)\right) \leftarrow P_{2}\left(\widehat{x}, \widehat{y}, \widehat{z}, x^{\prime}, y^{\prime}, z^{\prime}\right), P_{a}\left(\widehat{x^{\prime}}\right), P_{a}\left(\widehat{y^{\prime}}\right), P_{a}\left(\widehat{z^{\prime}}\right) . \\
P_{2}\left(\widehat{f(x)}, \widehat{g(y)}, \widehat{z}, x^{\prime}, y^{\prime}, z^{\prime}\right) \leftarrow P_{2}\left(\widehat{x}, \widehat{y}, \widehat{z}, h\left(x^{\prime}\right), i\left(y^{\prime}\right), z^{\prime}\right) \\
P_{2}(\widehat{x}, \widehat{y}, \widehat{z}, x, y, z) \leftarrow .\end{array}$ \\
\hline $\begin{array}{l}\text { A cycle is detected - removeCycles replaces the } \\
\text { blue clause by the red one. }\end{array}$ & $\begin{aligned} P_{2}\left(\widehat{f(x)}, \widehat{g(y)}, \widehat{z}, x^{\prime}, y^{\prime}, z^{\prime}\right) \leftarrow & P_{2}\left(\widehat{x}, \widehat{y}, \widehat{z_{1}}, h\left(x^{\prime}\right), i\left(y^{\prime}\right), z_{1}^{\prime}\right), \\
& P_{2}\left(\widehat{x_{1}}, \widehat{y_{1}}, \widehat{z}, h\left(x_{1}^{\prime}\right), i\left(y_{1}^{\prime}\right), z^{\prime}\right)\end{aligned}$ \\
\hline$P_{d}\left(d_{2}(f(h(x) \widehat{), g}(i(y)), z)) \leftarrow P_{1}(\widehat{x}, \widehat{y}, \widehat{z})\right.$ & $P_{d}\left(d_{2}(\widehat{x, y}, z)\right) \leftarrow P_{2}\left(\widehat{x}, \widehat{y}, \widehat{z}, x^{\prime}, y^{\prime}, z^{\prime}\right), P_{1}\left(\widehat{x^{\prime}}, \widehat{y^{\prime}}, \widehat{z^{\prime}}\right)$ \\
\hline $\begin{aligned} P_{d}(c(f(\widehat{x), g}(y))) \leftarrow & P_{2}\left(\widehat{x}, \widehat{y}, \widehat{z}, h\left(x^{\prime}\right), i\left(y^{\prime}\right), z^{\prime}\right) \\
& P_{a}\left(\widehat{x^{\prime}}\right), P_{a}\left(\widehat{y^{\prime}}\right)\end{aligned}$ & $\begin{array}{l}P_{3}(\widehat{f(x)}, \widehat{g(y)}) \leftarrow P_{2}\left(\widehat{x}, \widehat{y}, \widehat{z}, h\left(x^{\prime}\right), i\left(y^{\prime}\right), z^{\prime}\right), P_{a}\left(\widehat{x^{\prime}}\right), P_{a}\left(\widehat{y^{\prime}}\right) \\
P_{d}(\widehat{c(x, y)}) \leftarrow P_{3}(\widehat{x}, \widehat{y})\end{array}$ \\
\hline
\end{tabular}

Figure 4: Run of $\operatorname{comp}_{R}$ on Example 19

\section{Further Work}

Computing approximations more precise than regular ones is a first attempt towards a verification technique. However, there are at least two steps before considering our technique as a verification technique: 1) automatically handling the choices done during the normalization process and 2) extending to work with any rewrite system.

Concerning item 1, the quality of the approximation highly depends on the 
choice of the predicate symbol to be reused when predicate-limit is reached. On the other hand, the choice of generating function-symbols as output or as input is also crucial. Some automated heuristics will have to be designed in order to obtain well-customized approximations, for instance by extending the ideas of $[25]$.

Ongoing work tends to show that the linear restriction concerning the rewrite system can be tackled. A non right-linear rewrite system makes the computed S-CF program copying. Consequently, Theorem 2 does not hold anymore. To get rid of the right-linearity restriction, we are studying the transformation of a copying S-CF clause into non-copying ones that will generate an overapproximation. On the other hand, to get rid of the left-linearity restriction, we are studying a technique based on that of [9]. However, their method does not always terminate. We want to force termination thanks to an additional over-approximation.

\section{References}

[1] J. Kochems, C.-H. L. Ong, Improved Functional Flow and Reachability Analyses Using Indexed Linear Tree Grammars, in: RTA, Vol. 10 of LIPIcs, 2011, pp. 187-202.

[2] Y. Boichut, J. Chabin, P. Réty, Over-approximating descendants by synchronized tree languages, in: RTA, Vol. 21 of LIPIcs, 2013, pp. 128-142.

[3] Y. Boichut, B. Boyer, T. Genet, A. Legay, Equational Abstraction Refinement for Certified Tree Regular Model Checking, in: ICFEM, Vol. 7635 of LNCS, Springer, 2012, pp. 299-315.

[4] T. Genet, Decidable Approximations of Sets of Descendants and Sets of Normal Forms, in: RTA, Vol. 1379 of LNCS, Springer-Verlag, 1998, pp. 151-165.

[5] T. Genet, F. Klay, Rewriting for Cryptographic Protocol Verification, in: CADE, Vol. 1831 of LNAI, Springer-Verlag, 2000, pp. 271-290. 
[6] Y. Boichut, R. Courbis, P.-C. Héam, O. Kouchnarenko, Finer is Better: Abstraction Refinement for Rewriting Approximations, in: RTA, Vol. 5117 of LNCS, Springer, 2008, pp. 48-62.

[7] A. Bouajjani, P. Habermehl, A. Rogalewicz, T. Vojnar, Abstract Regular (Tree) Model Checking, STTT 14 (2) (2012) 167-191.

[8] Y. Boichut, P.-C. Héam, A Theoretical Limit for Safety Verification Techniques with Regular Fix-point Computations, IPL 108 (1) (2008) 1-2.

[9] S. Limet, G. Salzer, Proving Properties of Term Rewrite Systems via Logic Programs, in: RTA, Vol. 3091 of LNCS, Springer, 2004, pp. 170-184.

[10] P. Réty, J. Chabin, J. Chen, R-Unification thanks to SynchronizedContextfree Tree Languages, in: Workshop on Unification (UNIF), 2005.

[11] P. Réty, J. Chabin, J. Chen, Synchronized ContextFree Tree-tuple Languages, Tech. Rep. LIFO, RR-2006-13, University of Orleans / LIFO (2006).

[12] Y. Boichut, J. Chabin, P. Réty, Erratum of our RTA-13 paper, Tech. Rep. http://www.univ-orleans.fr/lifo/Members/rety/articles/PatchRTA13.pdf, LIFO, Université d'Orléans (2014).

[13] Y. Boichut, V. Pelletier, P. Réty, Synchronized tree languages for reachability in non-right-linear term rewrite systems, in: D. Lucanu (Ed.), Rewriting Logic and Its Applications - 11th International Workshop WRLA, Vol. 9942 of Lecture Notes in Computer Science, Springer, 2016, pp. 64-81.

[14] H. Comon, M. Dauchet, R. Gilleron, D. Lugiez, S. Tison, M. Tommasi, Tree Automata Techniques and Applications (TATA) (2007).

[15] J. Raoult, Rational Tree Relations, Bulletin of the Belgian Mathematical Society Simon Stevin 4 (1997) 149-176.

[16] P. Réty, Langages synchronisés d'arbres et applications. Habilitation Thesis (in French)., Tech. rep., LIFO, Université d'Orléans (June 2001). 
[17] W. C. Rounds, Context-free grammars on trees, in: P. C. Fischer, S. Ginsburg, M. A. Harrison (Eds.), STOC, ACM, 1969.

[18] J. Engelfriet, E. M. Schmidt, IO and OI (I), Journal of Computer and System Sciences 15 (3) (1977) 328 - 353. doi:http://dx.doi.org/10.1016/S00220000(77)80034-2.

[19] J. Engelfriet, E. M. Schmidt, IO and OI (II), Journal of Computer and System Sciences 16 (1) (1978) 67 - 99. doi:http://dx.doi.org/10.1016/00220000(78)90051-X.

[20] J. Engelfriet, L. Heyker, Context-free Hypergraph Grammars have the same Term-generating Power as Attribute Grammars, Acta Informatica 29.

[21] J. Engelfriet, J. Vereijken, Context-free Grammars and Concatenation of Graphs, Acta Informatica 34 (1997) 773-803.

[22] I. Durand, M. Sylvestre, Left-linear bounded trss are inverse recognizability preserving, in: RTA, Vol. 10 of LIPIcs, 2011, pp. 361-376.

[23] S. Limet, G. Salzer, Tree Tuple Languages from the Logic Programming Point of View, Journal of Automated Reasoning 37 (4) (2006) 323-349.

[24] V. Gouranton, P. Réty, H. Seidl, Synchronized Tree Languages Revisited and New Applications, in: FoSSaCS, Vol. 2030 of LNCS, Springer, 2001.

[25] T. Genet, V. Rusu, Equational Tree Automata Completion, Journal of Symbolic Computation, vol. 45, 2010. 